ARTICLE

https://doi.org/10.1038/s41467-019-09810-w

\title{
RUNX3 regulates cell cycle-dependent chromatin dynamics by functioning as a pioneer factor of the restriction-point
}

Jung-Won Lee ${ }^{1,5}$, Da-Mi Kim, ${ }^{1,}$, Ju-Won Jang ${ }^{1}$, Tae-Geun Park', Soo-Hyun Song ${ }^{1}$, You-Soub Lee ${ }^{1}$, Xin-Zi Chi', II Yeong Park ${ }^{2}$, Jin-Won Hyun ${ }^{3}$, Yoshiaki Ito ${ }^{4} \&$ Suk-Chul Bae (i) ${ }^{1}$

The cellular decision regarding whether to undergo proliferation or death is made at the restriction ( $R$ )-point, which is disrupted in nearly all tumors. The identity of the molecular mechanisms that govern the R-point decision is one of the fundamental issues in cell biology. We found that early after mitogenic stimulation, RUNX3 binds to its target loci, where it opens chromatin structure by sequential recruitment of Trithorax group proteins and cellcycle regulators to drive cells to the R-point. Soon after, RUNX3 closes these loci by recruiting Polycomb repressor complexes, causing the cell to pass through the R-point toward S phase. If the RAS signal is constitutively activated, RUNX3 inhibits cell cycle progression by maintaining R-point-associated genes in an open structure. Our results identify RUNX3 as a pioneer factor for the R-point and reveal the molecular mechanisms by which appropriate chromatin modifiers are selectively recruited to target loci for appropriate R-point decisions.

\footnotetext{
${ }^{1}$ Department of Biochemistry, School of Medicine, and Institute for Tumor Research, Chungbuk National University, Cheongju 28644, South Korea. ${ }^{2}$ College of Pharmacy, Chungbuk National University, Cheongju 361-763, South Korea. ${ }^{3}$ Department of Biochemistry, School of Medicine, Jeju National University, Jeju 63243, South Korea. ${ }^{4}$ Cancer Science Institute of Singapore, National University of Singapore, 14 Medical Drive, Singapore, Singapore $117599 .{ }^{5}$ These authors contributed equally: Jung-Won Lee, Da-Mi Kim. Correspondence and requests for materials should be addressed to S.-C.B. (email: scbae@chungbuk. ac.kr)
} 
$\mathrm{n}$ response to mitogenic stimulation, the cell makes a critical decision regarding whether to advance into late $G_{1}$, retreat into $G_{0}$, or undergo apoptosis. This decision occurs at the restriction (R)-point, and the associated decision-making machinery is perturbed in nearly all cancer cells ${ }^{1-3}$. The Rpoint decision-making process involves regulation of several hundred genes ${ }^{4}$. For silent genes to be induced, target sites within their regulatory regions must be bound de novo by transcription factors, which initiate their expression. The special transcription factors that have the capacity to associate with condensed chromatin independently of other factors and modulate chromatin accessibility are known as pioneer factors ${ }^{5-8}$.

To modulate chromatin accessibility and regulate gene transcription, pioneer factors require a complex network of other proteins, including coactivators, corepressors, histone-modifying complexes, chromatin-remodeling complexes, and the basal transcription machinery. For example, the Trithorax group (TrxG) and Polycomb group $(\mathrm{PcG})$ proteins establish histone modifications that activate and repress transcription, respectively. TrxG proteins can be broadly classified into two categories: histone modifiers ${ }^{9}$, and nucleosome remodelers ${ }^{10}$. The TrxG histone modifiers include mixed-lineage leukemia family members (MLLs), which methylate $\mathrm{H} 3$ at lysine 4 (H3K4-me3, -me2, and -me1), a mark that favors transcriptional activation. On the other hand, the TrxG nucleosome remodelers include the SWI-SNF complex, which facilitates binding of transcription factors and the basal transcription machinery. PcG complexes are classified into two categories: Polycomb repressor complex 1 and 2 (PRC1 and PRC2). Both complexes consist of multiple proteins: PRC1 contains BMI1 and ring finger protein 1 (RING1) or ring finger protein $2(\mathrm{RNF} 2)^{11}$, whereas PRC2 contains EED and enhancer of zeste homologs (EZH1 and EZH2) that trimethylate $\mathrm{H} 3$ at lysine 27 (H3K27-me3), a characteristic of inactive chromatin ${ }^{12}$. Via recruitment of these chromatin modulators, cells regulate signal-dependent gene expression at the correct target loci at the right time. The underlying mechanism, which represents one of the most fundamental issues in molecular biology, remains poorly understood.

The DNA-binding transcription factor RUNX3 plays pivotal roles in lineage determination ${ }^{13}$. Deletion of Runx3 in mouse lung results in development of lung adenomas and accelerates KRas-induced progression into adenocarcinomas (ADCs) ${ }^{14}$. In mouse embryonic fibroblasts, Runx3 deletion perturbs the Rpoint, leading to transformation ${ }^{4}$. Here, we demonstrate that RUNX3 is a pioneer factor of the R-point that plays a key role in sequential recruitment of TrxG and PcG proteins to target loci in a RAS signal-dependent manner, enabling an appropriate R-point decision.

\section{Results}

The RUNX3-BRD2-nucleosome complex recruits SWI/SNF and TFIID. The R-point decision is made $3-4 \mathrm{~h}$ after serum stimulation $^{15}$. Previously, we showed that the RUNX3-BRD2 complex forms $1-2 \mathrm{~h}$ after serum stimulation ${ }^{14}$, and that this complex contributes to the R-point decision by regulating hundreds of genes ${ }^{4}$. BRD2 contains two bromodomains (BD1 and $\mathrm{BD} 2$ ), each of which interacts with a distinct protein: BD1 binds RUNX3 acetylated at Lys-94 and Lys-171 ${ }^{14}$, whereas BD2 binds the acetylated histones H4K5-ac, H4K12-ac, and H3K14-ac ${ }^{16,17}$ (Fig. 1a). Notably, we detected interactions between p300, RUNX3, and H4K12-ac 1-2 h after mitogenic stimulation, as well as between BRD2, RUNX3, and H4K12-ac (Fig. 1b). The RUNX3-H4K12-ac interaction was markedly diminished by knockdown of BRD2 (see below). These results suggest that RUNX3 guides p300 to target loci, where it acetylates histones, and that BRD2 binds both acetylated RUNX3 and acetylated histones through its two bromodomains, prior to the R-point.

BRD2 interacts with the SWI/SNF and TFIID complexes through its C-terminal region ${ }^{17,18}$ (Fig. 1a), suggesting that RUNX3 interacts with these complexes through BRD2. We found that TAF1 (activating TAF), TAF7 (inhibitory TAF), and TBP formed a complex with BRD2 and RUNX3 $1 \mathrm{~h}$ after mitogenic stimulation (Fig. 1c). Soon thereafter, TAF7 dissociated from the complex (Fig. 1c), suggesting that TFIID is activated after the interaction with RUNX3-BRD2. After $4 \mathrm{~h}$, TAF1 and TBP also dissociated from RUNX3 (Fig. 1c). Similarly, BRG-1 and BAF155 (components of the SWI/SNF complex) also interacted with RUNX3 and BRD2 1-2 h after mitogenic stimulation and dissociated at $4 \mathrm{~h}$ (Fig. 1c). The interactions of SWI/SNF and TFIID complexes with the RUNX3-BRD2 complex were confirmed by the proximity ligation assay (PLA) (Supplementary Fig. 1a). Consistently, expression of R-point-associated proteins [p14 ${ }^{\mathrm{ARF}}$ (hereafter ARF), p53, and $\left.\mathrm{p} 21\right]^{4,14}$ was induced at the same time that RUNX3 interacted with BRD2, SWI/SNF, and TFIID (Supplementary Fig. 1b).

Knockdown of BRD2 revealed that H4K12-ac and the TFIID and SWI/SNF complexes interact with RUNX3 through BRD2 (Fig. 1d). SWI/SNF and TFIID interacted with transfected RUNX3 lacking the N-terminal 53 amino acid (aa) region (RX3- $\Delta \mathrm{Nt}$ ), but not with a RUNX3 mutant lacking Lys residues critical for the interaction with BRD2 (RUNX3-K94/171R)14 (Supplementary Fig. 1c). RUNX1, which shares high-sequence similarity with RUNX3, directly interacts with TAF1 though an interaction between acetylated Lys-43 of RUNX1 and the bromodomain of TAF $1{ }^{19}$. However, RUNX3 does not contain a lysine residue corresponding to Lys-43 of RUNX1 (Supplementary Fig. 1d), and therefore requires BRD2 for its interaction with SWI/SNF and TFIID.

Transient formation of the RUNX3-BRD2 complex was detected 1-2 $\mathrm{h}$ after treatment of EGF (an inducer of proliferation) (Supplementary Fig. 1e), but not after treatment with doxorubicin (an inducer of DNA damage) (Supplementary Fig. 1f), although both induced p53. These results suggest that transient formation of the RUNX3-BRD2 complex is R-pointspecific.

RUNX3 is a pioneer factor of the R-point. To elucidate the temporal order of the molecular interactions, we transfected cells with Myc-RUNX3 along with Flag-BRD2-WT, Flag-BRD2- $\Delta$ Ct (lacking aa 633-802 in the C-terminal region), Flag-BRD2- $\triangle \mathrm{BD} 1$ (lacking BD1), or Flag-BRD2- $\triangle \mathrm{BD} 2$ (lacking BD2). Analysis of subcellular localization revealed that all of the BRD2 deletion mutants localized to the nucleus (Supplementary Fig. 1g). Then, we performed immunoprecipitation/immunoblotting (IP/IB) to analyze interactions of the proteins $2 \mathrm{~h}$ after serum stimulation. As the RUNX3-p300 interaction is independent of BRD2 (Fig. 1d), it was not affected by expression of mutant BRD2 (Fig. 1e). Therefore, the RUNX3-p300 interaction must occur earlier than the RUNX-BRD2 interaction. Deletion of the Cterminal region of BRD2 (Flag-BRD2- $\Delta \mathrm{Ct}$ ) abolished the interactions of RUNX3 with TFIID and SWI/SNF without affecting the RUNX3-H4K12-ac interaction, whereas deletion of either $\mathrm{BD} 1$ or BD2 abolished the RUNX3-H4K12-ac interaction (Fig. 1e). These results confirmed that BRD2 simultaneously binds acetylated RUNX3 and acetylated H4 through BD1 and $\mathrm{BD} 2$, respectively. Notably, however, BRD2- $\triangle \mathrm{BD} 2$ failed to bridge between RUNX3, SWI/SNF, and TFIID (Fig. 1e). These results suggest that only BRD2 bound to both RUNX3 and histone (RUNX3-BRD2-nucleosome complex) can interact with the SWI/SNF and TFIID complexes. On the basis of these findings, 
a

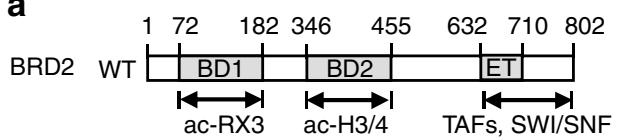

b Serum $\begin{array}{llllllll}0 & 1 & 2 & 4 & 8 & (\mathrm{~h}) & \mathrm{kDa}\end{array}$
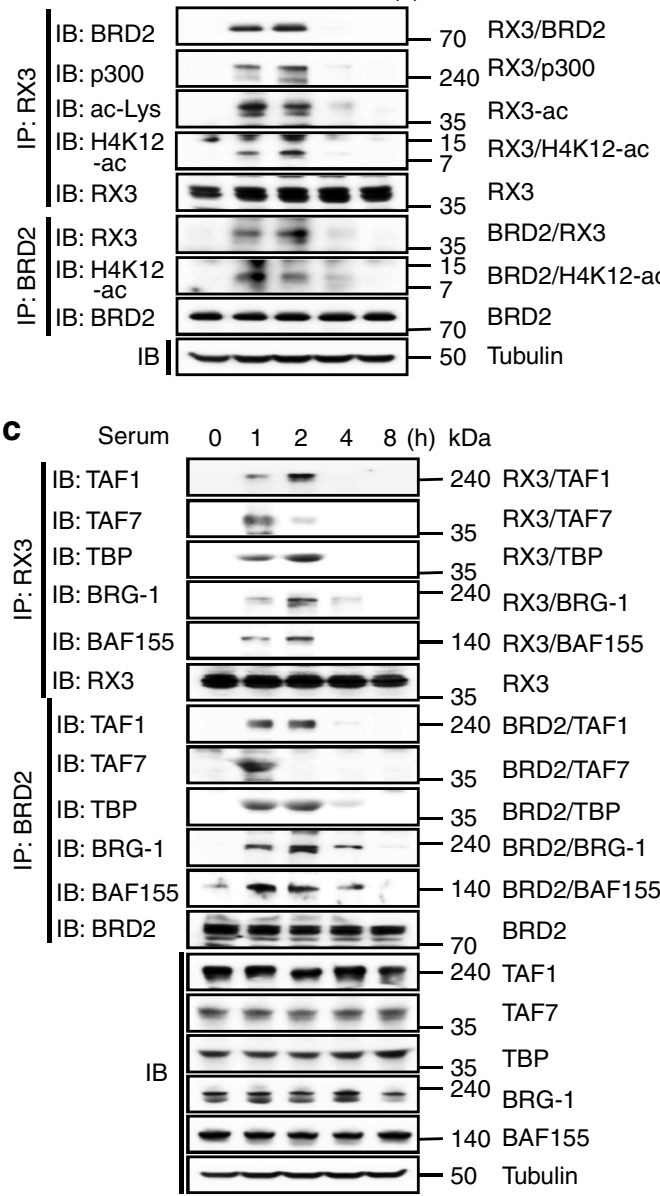

d

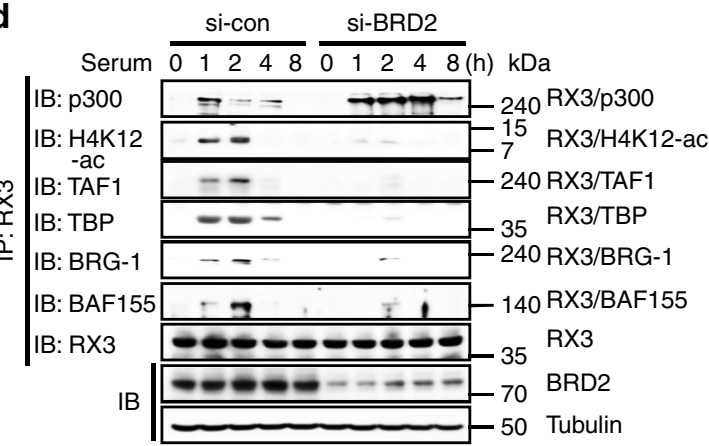

we conclude that RUNX3 on its binding site in an enhancer region facilitates p300-mediated histone acetylation around the locus, juxtaposes the promoter region via formation of the RUNX3-BRD2-H4K12-ac complex, and then recruits SWI/SNF and TFIID complexes to the locus via BRD2.

To confirm RUNX3-mediated recruitment of SWI/SNF and TFIID complexes to specific chromatin loci, we chose the ARF $(C D K N 2 A)$ locus as a model. ARF is a target of RUNX3 ${ }^{14}$ that is critical for the life and death of cells; thus, regulation of its expression could represent the R-point decision. The $A R F$ promoter contains a perfect match to the RUNX consensus binding site 1466 bases upstream of the transcription initiation

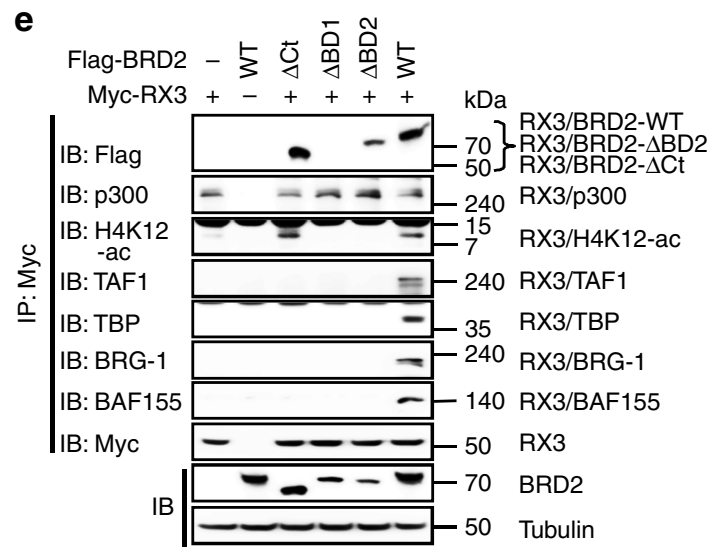

f

ARF-RX-D: 5'- AT CCAGGCAGGCT-3'

ARF-WT:5'- ATCCAGGCAGACCGCAGGCT-3'

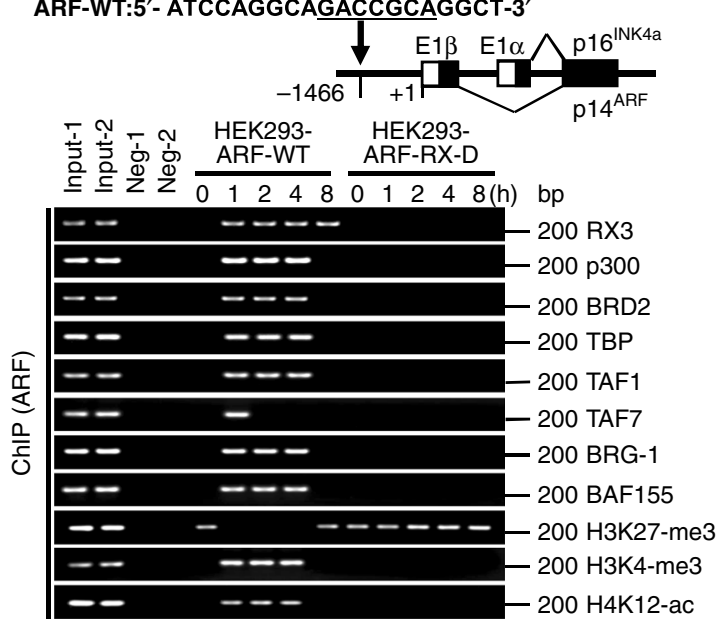

$\mathbf{g}$

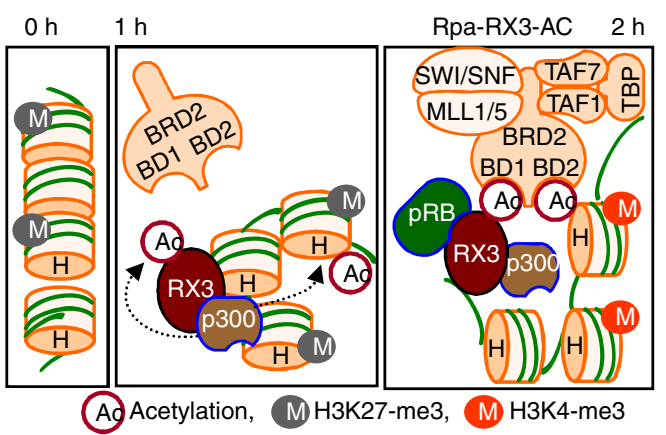

site $^{20}$. Using the CRISPR/Cas9 system, we deleted the RUNXbinding site in HEK293 cells to obtain HEK293-ARF-RX-D cells. Chromatin IP (ChIP) analysis revealed that in parental HEK293 cells (HEK293-ARF-WT), RUNX3 bound to the ARF locus $1 \mathrm{~h}$ after serum stimulation, and this interaction was maintained for $8 \mathrm{~h}$ (Fig. 1f). p300, BRD2, and components of SWI/SNF and TFIID were recruited to the locus $1 \mathrm{~h}$ after serum treatment, and the interaction was maintained for $4 \mathrm{~h}$ (except for TAF7, which dissociated after $2 \mathrm{~h}$ ) (Fig. 1f). By contrast, in HEK293ARF-RX-D cells, none of these proteins was recruited to the locus (Fig. 1f). These results indicate that binding of RUNX3 to the RUNX consensus site is critical for recruitment of the 
Fig. 1 The RUNX3-BRD2-nucleosome complex recruits SWI/SNF and TFIID. a Schematic diagram of BRD2 structure and interacting proteins. BD1 interacts with RUNX3 acetylated at Lys-94 and Lys-171; BD2 interacts with acetylated histones H4K4-ac, H4K12-ac, and H3K14-ac; and the C-terminal region interacts with the TFIID and SWI/SNF complexes. b, c HEK293 cells were serum-starved for $24 \mathrm{~h}$, and then stimulated with $10 \%$ serum. Cells were harvested at the indicated time points, and the levels of the indicated proteins were measured by IP and IB. The time-dependent interactions were measured by IP and IB. d HEK293 cells were treated with control siRNA (si-con) or BRD2-specific siRNA (si-BRD2), serum-starved for 24 h, and then stimulated with $10 \%$ serum for the indicated durations. The time-dependent interactions between the proteins were measured by IP and IB. e HEK293 cells were transfected with Myc-RUNX3, Flag-BRD2-WT, Flag-BRD2- $\Delta$ Ct (lacking C-terminal aa 633-802), Flag-BRD2- $\Delta$ BD1 (lacking BD1), or Flag-BRD2- $\Delta$ BD2 (lacking BD2). Cells were serum-starved for $24 \mathrm{~h}$, and then stimulated with $10 \%$ serum. Cells were harvested after $2 \mathrm{~h}$, and the interactions of the proteins were measured by IP and IB. $\mathbf{f}$ The RUNX3-binding site (GACCGCA) in the ARF enhancer region (ntd -1466) was deleted in HEK293 cells by the CRISPR/ Cas9 method to obtain the HEK293-ARF-RX-D cell line. Deletion of the RUNX3-binding site was confirmed by nucleotide sequencing. Wild-type HEK293 cells (HEK293-ARF-WT) and HEK293-ARF-RX-D cells were serum-starved for $24 \mathrm{~h}$. The cells were then treated with $10 \%$ serum, and the binding of the indicated proteins to the ARF promoter was measured by ChIP at the indicated time points. One-thirtieth of the lysates were PCR-amplified as input samples. $\mathbf{g}$ Schematic illustration of sequential molecular events at RUNX3 target loci during R-point regulation. RUNX3 binds to condensed chromatin marked by H3K27-me3 (inhibitory mark). p300 recruited to the loci acetylates RUNX3 and histones. Then, BRD2 binds both acetylated RUNX3 and acetylated histone through its two bromodomains. At $1 \mathrm{~h}$ after serum stimulation, SWI/SNF and TFIID are recruited to the loci through the C-terminal region of BRD2 to form Rpa-RX3-AC, and H3K27-me3 is replaced by H3K4-me3 (activating mark)

chromatin-remodeling complex and basal transcriptional machinery to its target locus.

In HEK293-ARF-WT cells, H3K27-me3 (a repressive histone modification) was enriched within the $A R F$ promoter region prior to serum stimulation ( $0 \mathrm{~h})$ (Fig. 1f). Notably, H3K27-me3 was replaced by $\mathrm{H} 3 \mathrm{~K} 4-\mathrm{me} 3$ (an activating histone modification) 1-4 $\mathrm{h}$ after serum stimulation, whereas H3K27-me3 was restored $8 \mathrm{~h}$ later (Fig. 1f). Similarly, H4K12-ac (an activating histone modification) was detected $1-4 \mathrm{~h}$ after stimulation, but then disappeared (Fig. 1f). By contrast, in HEK293-ARF-RX-D cells, the H3K27-me3 modification was maintained, and H4K12 was not acetylated for a long time after serum stimulation (Fig. 1f). Consistently, ARF and p53 were induced 1-2 h after serum stimulation in HEK293-ARF-WT cells, but not in HEK293-ARFRX-D cells (Supplementary Fig. 1h). These results demonstrate that RUNX3 associates with its binding site and opens and activates the chromatin structure of target loci $1-2 \mathrm{~h}$ after serum stimulation, and then subsequently closes the loci, indicating that RUNX3 is a pioneer factor of the R-point. We named the RUNX3-containing complex formed before the R-point as the R-point-associated RUNX3-containing activator complex (Rpa-RX3-AC) (Fig. 1g).

In HEK293-ARF-WT cells, BRD2, SWI/SNF, and TFIID continued to be associated with the ARF locus up to $4 \mathrm{~h}$ after stimulation (Fig. 1f), but these proteins interacted with RUNX3 for only up to $2 \mathrm{~h}$ (Fig. $1 \mathrm{~b}, \mathrm{c}$ ). These results suggest that BRD2 remains bound to the $A R F$ locus for a while even after dissociation from RUNX3, most likely through interactions with histones and other DNA-binding proteins recruited to open chromatin. The sequence of molecular events is summarized in Fig. 1g. This model suggests that the enhancer interacts with the promoter through Rpa-RX3-AC during the R-point (Fig. 1g). The enhancer-promoter interaction on the $A R F$ locus $2 \mathrm{~h}$ after serum stimulation was confirmed by the chromosome conformation capture assay (3 $\mathrm{C}$ assay ${ }^{21}$ ) (Supplementary Fig. 2).

RUNX3 also recruits MLL1/5, PRC1, and PRC2. To understand how the RUNX3-BRD2 complex controls histone modifications, we performed yeast two-hybrid screening using the C-terminal region of BRD2 (aa 450-802) as bait. The screen identified MLL5 and RNF2, which play opposing roles in chromatin dynamics (Fig. 2a): MLL5 is a TrxG histone modifier that contributes to chromatin activation, whereas RNF2 is a component of the PRC1 complex, which inactivates chromatin.

Analysis of the interactions among BRD2 and MLL family members revealed that BRD2 interacted with MLL1 and MLL5 1-2 $\mathrm{h}$ after serum stimulation (Fig. 2b). These proteins also interacted with RUNX3 at the same time points (Fig. 2b). The physical interactions between MLL1/5 and RUNX3 at $2 \mathrm{~h}$ after serum stimulation were confirmed by the PLA (Fig. 2c). We confirmed that the RUNX3-MLL1/5 interactions are mediated by BRD2 (Supplementary Fig. 3). A genetic interaction between Lozenge (a Drosophila homolog of the RUNX family genes) and Trithorax (a Drosophila homolog of the MLL family genes) was also observed during fly eye development (Fig. 2d).

ChIP analysis revealed that MLL1/5 bound to the $A R F$ locus 1-4 $\mathrm{h}$ after stimulation, and H3K4-me3 was enriched at the locus at these time points (Fig. 2e). These results suggest that MLL1/5 recruited to chromatin through the interaction with the RUNX3-BRD2 complex forms activating histone modifications. Therefore, MLL1/5 are additional components of Rpa-RX3-AC.

Analysis of interactions among BRD2 and components of PRC1 revealed that BRD2 interacted with RNF2 and BMI1 4-8 h after serum stimulation (Fig. 2b). At that time, RUNX3 associated with Cyclin D1 and HDAC4 instead of BRD2 (Fig. 2b). EED and EZH2 (components of PRC2) associated with RUNX3 $8 \mathrm{~h}$ after stimulation (Fig. 2b). ChIP analysis revealed that Cyclin D1, HDAC4, and EZH2 were bound to chromatin after the R-point, at a time when $\mathrm{H} 4 \mathrm{~K} 12-\mathrm{ac}$ was absent and $\mathrm{H} 3 \mathrm{~K} 27-\mathrm{me} 3$ was enriched at the locus (Fig. 2e). After $4 \mathrm{~h}, \mathrm{RNF} 2$ briefly interacted with RUNX3 and BRD2 (Fig. 2b) and was recruited to the target locus (Fig. 2e), suggesting that PRC1 and Rpa-RX3-AC form a transient complex immediately before the latter complex is destroyed (between 2 and $4 \mathrm{~h}$ after mitogen stimulation). We named the transient assembly as the R-point-associated RUNX3containing transient complex (Rpa-RX3-TR) (Fig. 2f).

At $4-8 \mathrm{~h}$ after mitogen stimulation, RUNX3 and BRD2 existed in separate complexes: RUNX3 formed a complex with Cyclin D1, HDAC4, and PRC2 (Fig. 2b), which remained bound to target chromatin loci (Fig. 2e), whereas BRD2 formed the BRD2-PRC1 complex (Fig. 2b), which was released from the loci (Fig. 2e). As the RUNX3-Cyclin D1-HDAC4-PRC2 complex inactivates chromatin, we named it the R-point-associated RUNX3-containing repressor complex (Rpa-RX3-RE) (Fig. 2f).

The PRC1-Cyclin D1-HDAC4 complex interacts with RpaRX3-AC. We next investigated how Rpa-RX3-TR is formed. Cyclin D1 is induced $2 \mathrm{~h}$ after serum stimulation, and the induction of Cyclin D1 is independent of RUNX3 ${ }^{14}$. IP/IB analysis revealed that the RNF2-Cyclin D1 interaction occurred $2 \mathrm{~h}$ after serum stimulation and gradually weakened thereafter (Fig. 3a). The RNF2-HDAC4 interaction was detected only $4 \mathrm{~h}$ after stimulation (Fig. 3a), whereas the HDAC4-Cyclin D1 interaction occurred $4 \mathrm{~h}$ after simulation, and gradually 
a

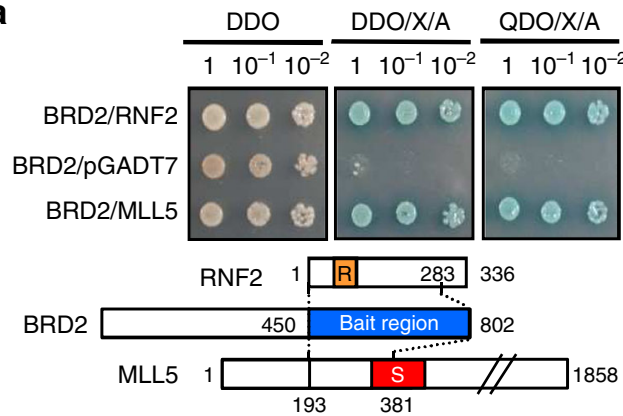

b $\quad$ Serum $\begin{array}{lllllll}0 & 1 & 2 & 4 & 8(\mathrm{~h}) & \mathrm{kDa}\end{array}$

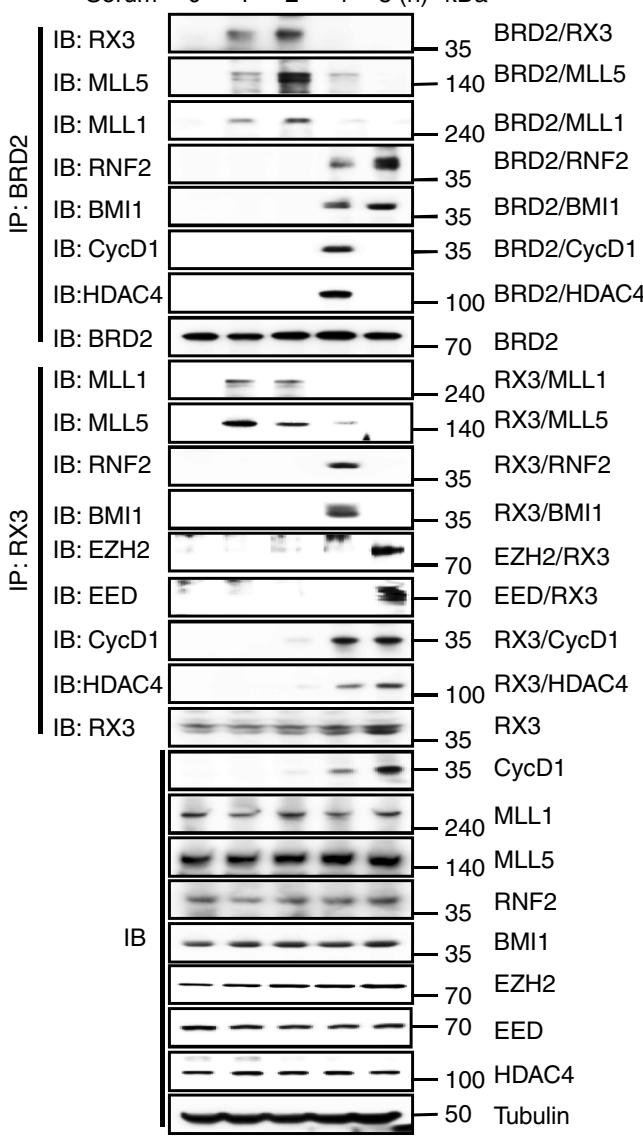

c

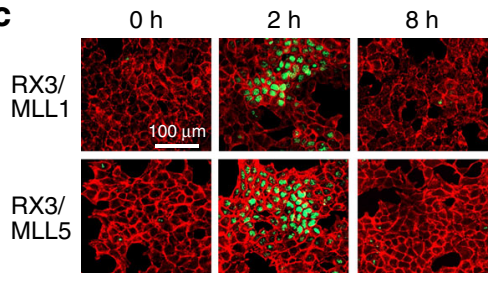

d
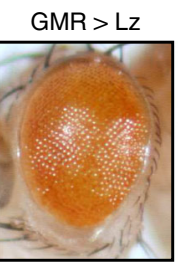

GMR $>$ Trx

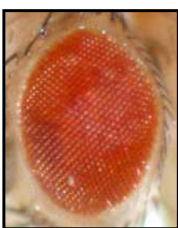

GMR $>$ Lz + Trx

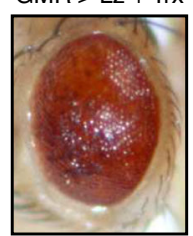

e

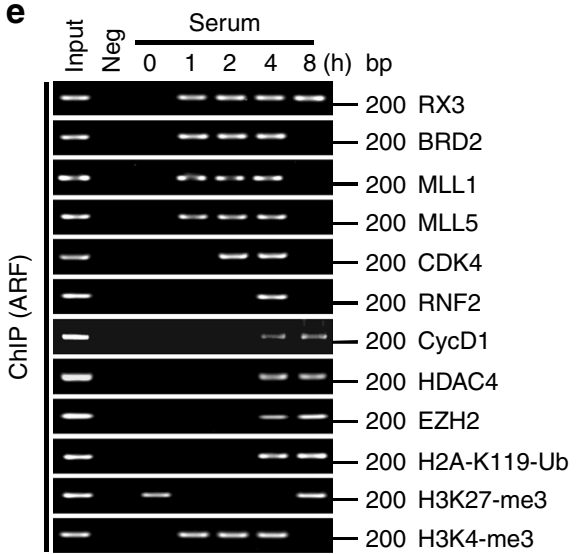

f

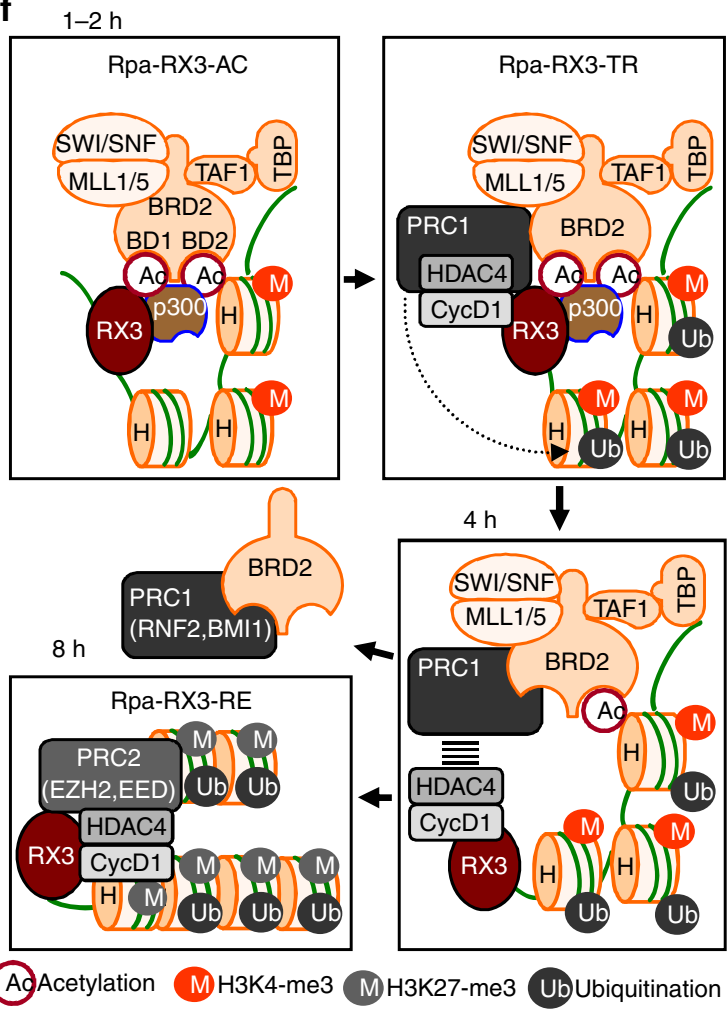

strengthened thereafter (Fig. 3a). These results suggest that PRC1 interacts with Cyclin D1 $2 \mathrm{~h}$ after stimulation and subsequently matures to the PRC1-Cyclin D1-HDAC4 complex. Formation of the PRC1-Cyclin D1-HDAC4 complex $4 \mathrm{~h}$ after stimulation was confirmed by transfection followed by IP/IB analysis (Fig. 3b), and the direct interactions between the proteins were confirmed by IP/IB with in vitro translated proteins (Fig. 3c, d). The results of domain mapping analysis for the interactions are summarized in Fig. 3e and Supplementary Fig. 4a-f.

PRC1, Cyclin D1, and HDAC4 were recruited to target loci $4 \mathrm{~h}$ after serum stimulation (Fig. 2e). Notably, small-interfering
RNA (siRNA)-mediated knockdown of HDAC4 inhibited dissociation of RUNX3-BRD2 (Fig. 3f). Similarly, knockdown of either RNF2 or Cyclin D1 diminished the RUNX3-Cyclin D1, RUNX3-HDAC4, and RNF2-BRD2 interactions and effectively inhibited dissociation of RUNX3-BRD2 (Fig. 3g). These results suggest that the PRC1-Cyclin D1-HDAC4 complex, rather than any of the individual components, interacts with Rpa-RX3-AC to form Rpa-RX3-TR. As p300-mediated RUNX3 acetylation is effectively removed by HDAC4 ${ }^{22}$, HDAC4, a component of RpaRX3-TR, may play a key role in deacetylation of RUNX3, causing it to release BRD2 and other BRD2-associated proteins. 
Fig. 2 RUNX3 sequentially recruits TrxG and PcG complexes. a Yeast two-hybrid screening using Gal4-BRD2 (aa 450-802) as bait identified RNF2 and MLL5 as BRD2-binding proteins (see STAR methods). DDO =SD-Leu/-Trp, DDO/X/A =SD-Leu/-Trp/X- $\alpha$-gal/ABA, QDO/X/A =SD-Leu/-Trp/-His/Ade/X- $\alpha$-gal/ ABA medium. Selective colonies were identified by DNA sequencing. $\mathbf{b}$ HEK293 cells were serum-starved for $24 \mathrm{~h}$, and then stimulated with $10 \%$ serum. Cells were harvested at the indicated time points, and the time-dependent interactions between RUNX3, BRD2, Cyclin D1, MLL1, MLL5, RNF2, BMI1, EZH2, EED, and HDAC4 were measured by IP and IB. c PLA showing RUNX3-MLL1 and RUNX3-MLL5 at the indicated time points after serum stimulation. Green fluorescence indicates association of the indicated proteins. F-actin was stained (red) to visualize the cytoplasmic compartment. d Microscopy images of transgenic fly eyes. Lozenge is a Drosophila homolog of the RUNX genes. Glass multimer reporter (GMR)-Gal4 promotes eye-specific expression of UAS-inserted genes. GMR-driven Lozenge overexpression (GMR-Gal4/ + ;UAS-Lozenge (Iz) $\rightarrow$ GMR $>$ Lz) or GMR-driven Trithorax (Trx) overexpression (GMR-Gal4/+; TrxG14137 $\rightarrow$ GMR > Trx) conferred weak rough phenotypes. However, GMR-driven overexpression of both Lz and Trx $(G M R>L z+T r x)$ resulted in a severe defective eye phenotype with loss of external ommatidial facets. e HEK293 cells were serum-starved for $24 \mathrm{~h}$, and then stimulated with 10\% serum. The binding of RUNX3, BRD2, MLL1, MLL5, CDK4, RNF2, Cyclin D1, HDAC4, EZH2, H2A-K119-Ub, H3K27-me3, and $\mathrm{H} 3 \mathrm{~K} 4-\mathrm{me} 3$ to the ARF promoter was measured by ChIP at the indicated time points. One-thirtieth of the lysates were PCR-amplified as input samples. f Schematic illustration of the R-point transition. At $1 \mathrm{~h}$ after serum stimulation, RUNX3 associates with various proteins, including p300, BRD2, H4K12-ac, SWI/SNF, TFIID, and MLL1/5, to form Rpa-RX3-AC. Between 2 and $4 \mathrm{~h}$ after serum stimulation, Rpa-RX3-AC interacts with PRC1-CyclinD1-HDAC4 to from a transient complex, Rpa-RX3-TR. Subsequently, Rpa-RX3-TR is destroyed (at $4 \mathrm{~h}$ ) to form Rpa-RX3-RE (at $8 \mathrm{~h}$ )

Inactivation of chromatin is associated with HDAC-mediated histone deacetylation and RNF2-mediated $\mathrm{H} 2 \mathrm{~A}$ ubiquitination at Lys-119 (H2A-K119-Ub) ${ }^{11}$. Consistently, H4K12 acetylation was reduced (Fig. 1f) and H2A ubiquitination at Lys-119 (H2AK119-Ub) was enriched at the $A R F$ locus $4-8 \mathrm{~h}$ after stimulation (Fig. 2e). These results demonstrate that the PRC1-Cyclin D1-HDAC4 complex binds to Rpa-RX3-AC, forms Rpa-RX3$\mathrm{TR}$, and then contributes to inactivation of chromatin at target loci by deacetylating H4K12 and ubiquitinating H2A (Fig. 3h).

\section{Association of CDK4 and Cyclin D1 leads to formation of Rpa-} RX3-TR. Previously, we showed that hypo-phosphorylated pRB interacts with RUNX3-BRD2 and contributes to R-point commitment ${ }^{4}$. E2F1 also interacted with RUNX3 1-2 h after stimulation (Fig. 4a). The pRB-E2F1 complex was released from RUNX3 when pRB was phosphorylated at Ser-795 by Cyclin D1-CDK4/6 (Fig. 4a). These results suggest that CDK4 approaches Rpa-RX3-AC to phosphorylate pRB. IP/IB analysis revealed that CDK4 interacted with RUNX3 $2 \mathrm{~h}$ afterward, and that the interaction weakened thereafter (Fig. 4a). CDK4 was also bound to the ARF locus while it interacted with RUNX3 (Fig. 2e). The physical interaction between RUNX3 and CDK4 $2 \mathrm{~h}$ after serum stimulation was confirmed by the PLA (Fig. 4b). These results demonstrate that CDK4, along with $\mathrm{pRB}$ and E2F1, which play key roles in cell-cycle regulation, is a component of Rpa-RX3-AC.

Although pRB and CDK4 were brought together within Rpa$\mathrm{RX} 3-\mathrm{AC} 2 \mathrm{~h}$ after serum stimulation, CDK4-mediated $\mathrm{pRB}$ phosphorylation occurred only $4 \mathrm{~h}$ after stimulation (Fig. 4a). Analysis of the timing of interactions among CDK4 and its binding proteins revealed that CDK4 interacted with p16 $1 \mathrm{~h}$ after stimulation, and then replaced p16 with p21 at $2 \mathrm{~h}$ (Fig. 4a). p21 facilitates the association of Cyclin D1 and CDK $4^{3,23,24}$. However, Cyclin D1, which was induced $2 \mathrm{~h}$ after stimulation, interacted with RNF2, but not with p21-bound CDK4 at that time point (Figs. 3a and 4a). The Cyclin D1-CDK4 interaction was detected after Rpa-RX3-TR formed (at $4 \mathrm{~h}$ ) (Fig. 4a). Notably, knockdown of CDK4 markedly diminished the interactions of RUNX3 with Cyclin D1 and HDAC4 (Fig. 4c). Consequently, the RUNX3-BRD2 interaction and ARF expression were maintained for up to $8 \mathrm{~h}$ (Fig. 4c). These results suggest that CDK4 of RpaRX3-AC and Cyclin D1 of PRC1-Cyclin D1-HDAC4 provide docking sites for the interaction of the two complexes, enabling formation of Rpa-RX3-TR.

Cyclin D1 and CDK4 contribute to the R-point transition. We then investigated the role of CDK4 in R-point transition. Pharmacological inhibition of CDK4 activity maintained Rpa-RX3-AC and prolonged ARF expression for up to $8 \mathrm{~h}$ (Fig. 4d). These results suggest that activation of CDK4 by the Cyclin D1-CDK4 interaction within Rpa-RX3-AC triggers the R-point transition.

Exogenously expressed Myc-RUNX3 and Flag-CDK4 also interacted $2-4 \mathrm{~h}$ after serum stimulation, but dissociated thereafter (Supplementary Fig. 5a). Interestingly, a kinase-dead CDK4 mutant (Flag-CDK4-K35M) bound to RUNX3 and did not dissociate for a long time after serum stimulation (Supplementary Fig. 5a). These results demonstrate that CDK4 kinase activity is required for dissociation of CDK4 from RUNX3.

CDK4 is known to phosphorylate RUNX3 at Ser-356 25 . We raised rabbit polyclonal anti-serum against synthetic RUNX3 peptide phosphorylated at Ser-356 (Supplementary Fig. 5b). The anti-serum specifically recognized only RUNX3 phosphorylated at Ser-356, but not RUNX1, RUNX2, or RUNX3-S356A (Supplementary Fig. 5c). This anti-serum detected phosphorylation of endogenous RUNX3 contemporaneously with the RUNX3-CDK4 interaction (2-4h after serum stimulation) (Fig. 4e). RUNX3 phosphorylation was markedly diminished by knockdown of CDK4 (Fig. 4c). Notably, the interaction between exogenously expressed Myc-RUNX3-S356A and BRD2 and expression of ARF were maintained up to $8 \mathrm{~h}$ after serum stimulation (Fig. 4f). However, a phosphorylation-mimic mutation of RUNX3 (Myc-RUNX3-S356E) did not affect association/ dissociation of RUNX3 and BRD2 (Fig. 4g). These results suggest that the CDK4-dependent RUNX3 phosphorylation at S356 is required, but not sufficient, for the release of BRD2 from RUNX3. The sequence of molecular events are summarized in Fig. $4 \mathrm{~h}$.

Multiple pathways contribute to the R-point transition. Treatment with MEK1 inhibitor abolished the BRD2-RUNX3 and p300-RUNX3 interactions (Fig. 5a). Consistent with this, p300-RUNX3 interaction was promoted by ectopic expression of constitutively activated MEK1 (MEK1-CA), but inhibited by kinase-dead MEK1 (MEK1-KD) (Supplementary Fig. 6a). These results demonstrate that the RAS-MEK signaling pathway stimulates formation of Rpa-RX3-AC.

The Cyclin D1-CDK4 interaction occurred $4 \mathrm{~h}$ after serum stimulation, and Cyclin D1-CDK4-dependent $\mathrm{pRB}$ phosphorylation occurred at the same time point (Fig. 4a). Notably, however, CDK4-dependent RUNX3 phosphorylation occurred $2 \mathrm{~h}$ after serum stimulation (Fig. 4c, e), earlier than the Cyclin D1-CDK4 interaction (Fig. 4a). Knockdown of CyclinD1 effectively reduced pRB phosphorylation (S795), but did not affect RUNX3 phosphorylation (S356) (Fig. 5b). These results suggest that CDK4 is activated in a CyclinD1-independent manner to promote RUNX3 phosphorylation. 
a

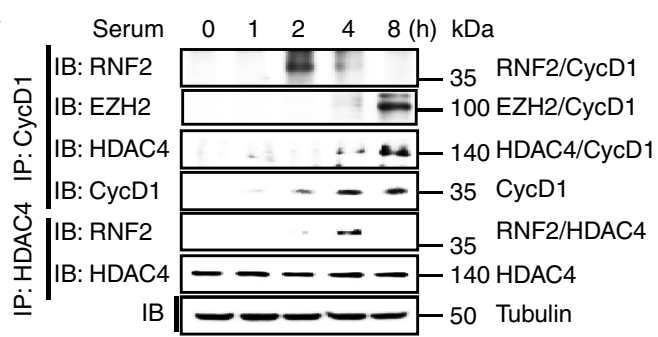

b

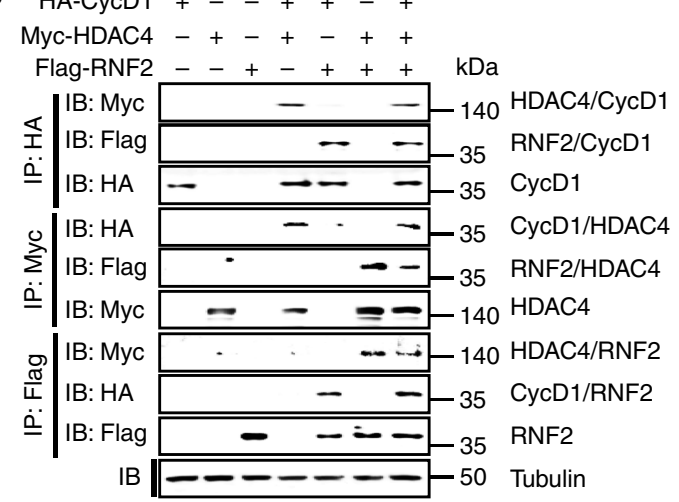

C $\mathrm{HA}-\mathrm{CyCD} 1+-+$

Myc-RNF2 - + $+\mathrm{kDa}$

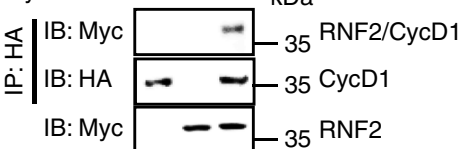

$\mathbf{d}_{\text {Myc-HDAC4 } 4+-+}$

$\mathrm{HA}-\mathrm{CyCD} 1+++\mathrm{kDa}$

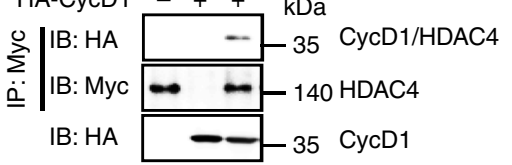

h

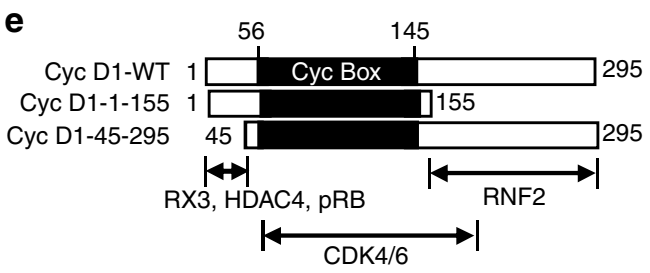

f

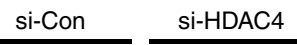

Serum $\begin{array}{lllllllllll}0 & 1 & 2 & 4 & 8 & 0 & 1 & 2 & 4 & 8 & (h) \\ k D a\end{array}$

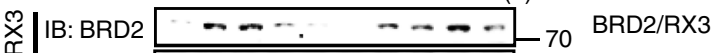

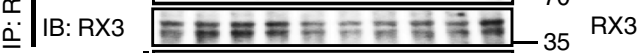

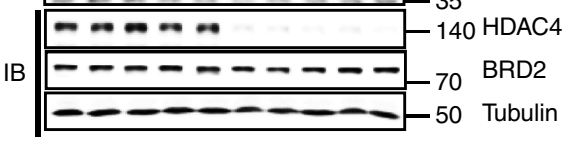

g

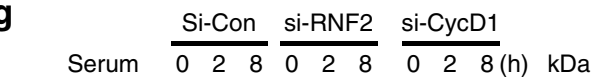
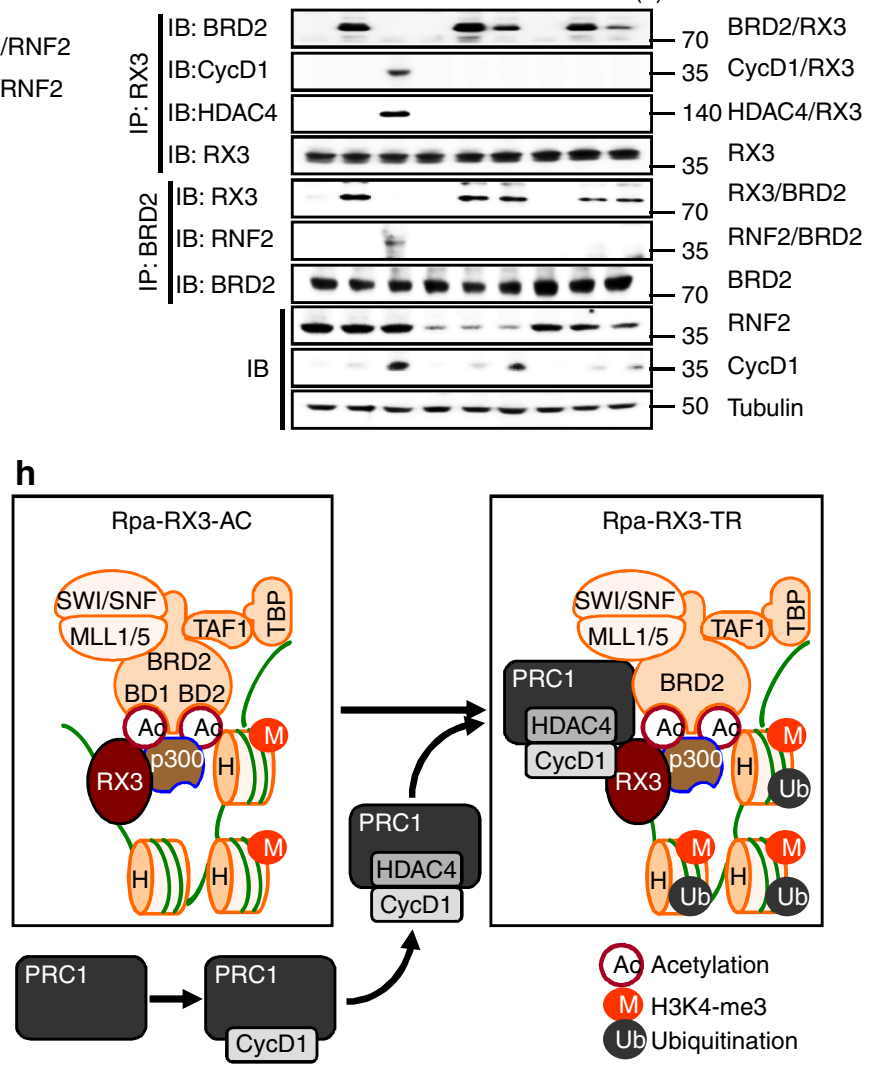

Fig. 3 Formation of the PRC1-CyclinD1-HDAC4 complex. a HEK293 cells were serum-starved for $24 \mathrm{~h}$, and then stimulated with $10 \%$ serum. Cells were harvested at the indicated time points. Time-dependent formation of the RNF2-Cyclin D1, EZH2-Cyclin D1, HDAC4-Cyclin D1, and RNF2-HDAC4 complexes was measured by IP and IB. b HEK293 cells were transfected with HA-Cyclin D1, Myc-HDAC4, and Flag-RNF2, and the interactions between the proteins were measured by IP and IB. c, d HA-Cyclin D1, Myc-RNF2, and Myc-HDAC4 were translated in vitro and the interactions among the proteins were measured by IP and IB. e Regions of Cyclin D1 required for the interaction with RUNX3, RNF2, and HDAC4 are summarized. Cyclin D1 regions known to interact with pRB and CDK4/6 are also indicated. Cyc Box $=$ Cyclin Box. $\mathbf{f H E K} 293$ cells were treated with control or HDAC4-specific siRNA (si-con or si-HDAC4), serum-starved for $24 \mathrm{~h}$, and then stimulated with serum for the indicated durations. Time-dependent formation of the BRD2-RUNX3 complex was measured by IP and IB. $\mathbf{g}$ HEK293 cells were treated with control, RNF2-specific, or Cyclin D1-specific siRNA (si-con, si-RNF2, or si-CycD1), serumstarved for $24 \mathrm{~h}$, and then stimulated with serum for the indicated durations. Time-dependent formation of the BRD2-RUNX3, Cyclin D1-RUNX3, HDAC4-RUNX3, and RNF2-BRD2 complexes was measured by IP and IB. h Schematic illustration of the process of Rpa-RX3-TR formation. Cyclin D1, which is induced $2 \mathrm{~h}$ after serum stimulation, interacts with PRC1 (containing RNF2) and matures into the PRC1-CyclinD1-HDAC4 complex. The PRC1-CyclinD1-HDAC4 complex then interacts with Rpa-RX3-AC to form Rpa-RX3-TR 

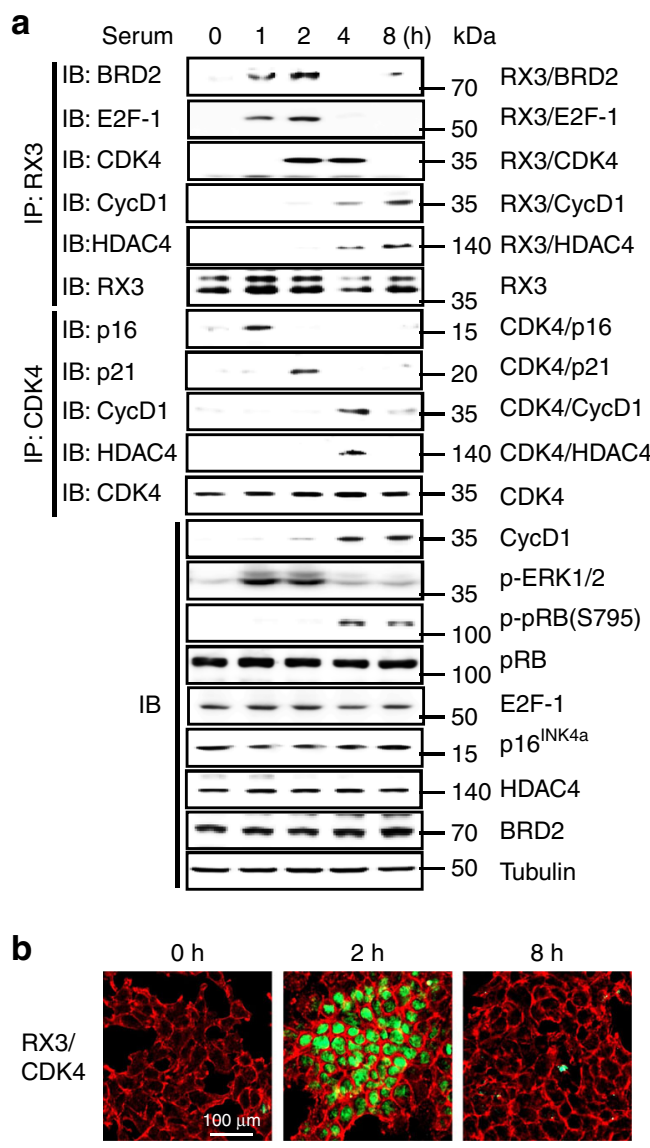

C
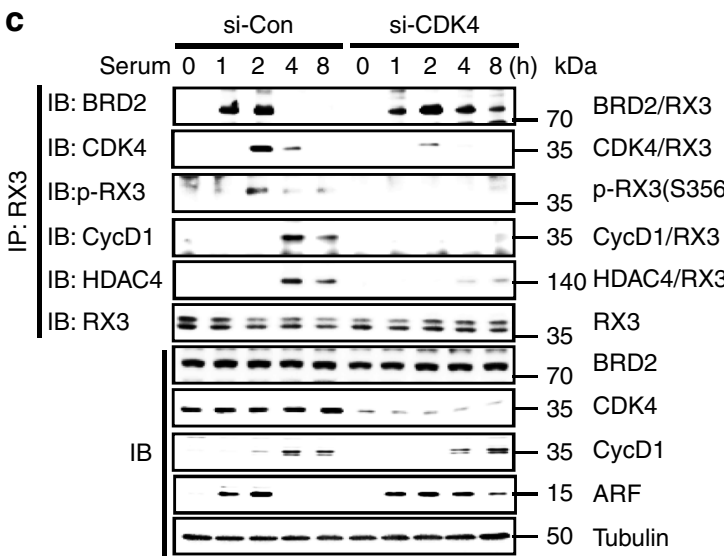

Activation of CDK4 depends upon CDK-activating kinase (CAK) activity $^{26}$. JNK was identified as one of the CAKs that phosphorylates CDK4 at T172 27 . We found that JNK phosphorylates CDK4 (at T172) $2 \mathrm{~h}$ after serum stimulation (Fig. 5c). Pharmacological inhibition of JNK activity markedly reduced RUNX3 phosphorylation at Ser-356 and maintained Rpa-RX3AC for up to $8 \mathrm{~h}$ (Fig. 5d). Similarly, siRNA-mediated knockdown of JNK also decreased RUNX3 phosphorylation at Ser-356 and maintained Rpa-RX3-AC (Fig. 5e). Exogenously expressed FlagCDK4-WT phosphorylated Myc-RUNX3 2-4h after serum stimulation. However, Flag-CDK4-T172A (lacking the residue phosphorylated by JNK) failed to phosphorylate RUNX3 at d

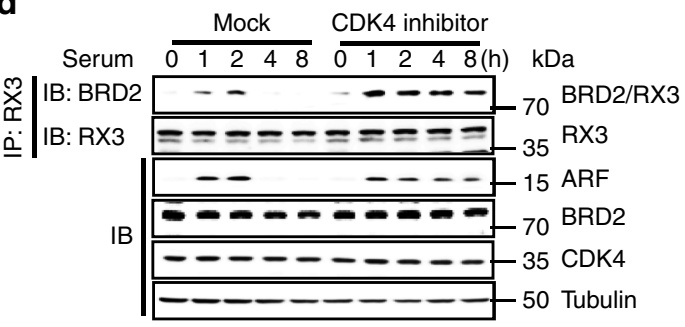

e

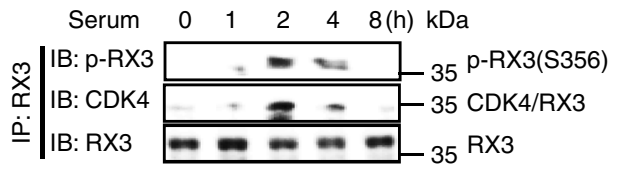

f
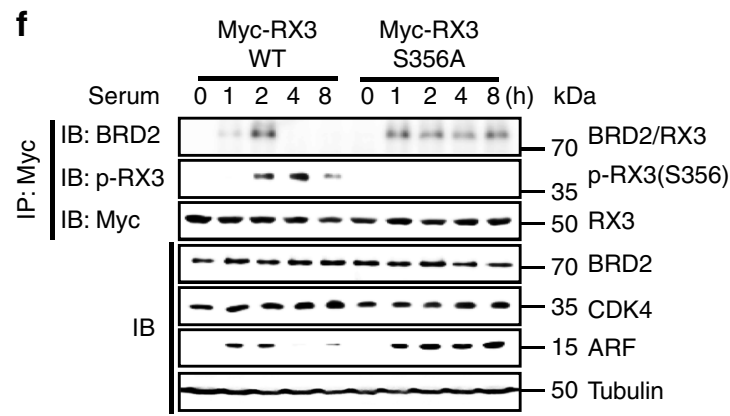

g

Myc-RX3 Myc-RX3

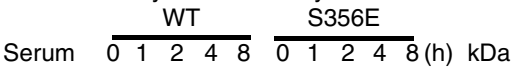

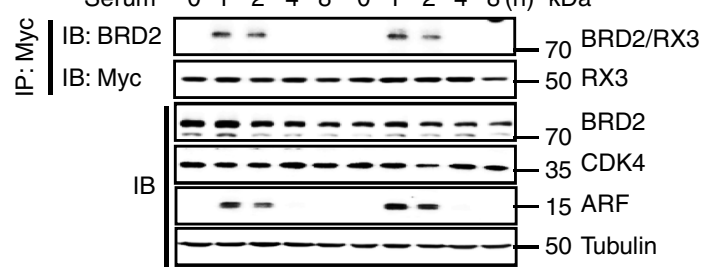

h

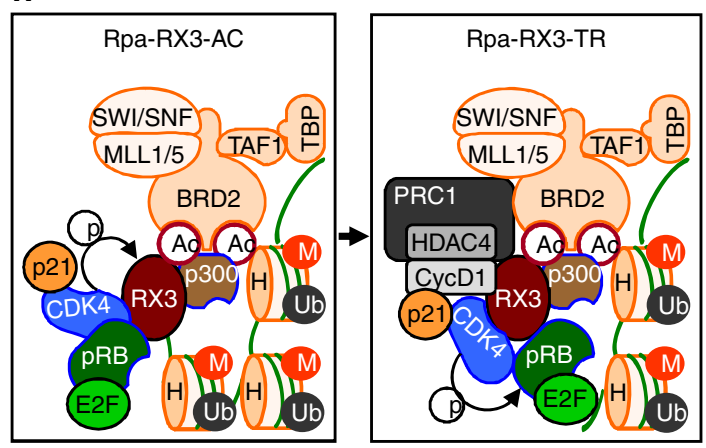

Ser-356 (Fig. 5f). Flag-CDK4-T172A bound to RUNX3 even earlier, and did not dissociate for a long time after serum stimulation (Fig. 5f). Consistently, Flag-CDK4-T172A maintained Rpa-RX3-AC and prolonged ARF expression for up to $8 \mathrm{~h}$ (Fig. 5f). These results demonstrate that the JNK pathway also contributes to the R-point transition by activating CDK4.

Transcription and translation of Cyclin D1, which plays a key role in formation of Rpa-RX3-TR, are stimulated through the RAS-RAF and RAS-PI3K pathways, respectively ${ }^{3}$. As expected, knockdown of the PI3K catalytic subunit (PIK3CA, encoding p110a) decreased the level of Cyclin D1 and maintained RpaRX3-AC and prolonged ARF expression for up to $8 \mathrm{~h}$ (Fig. $5 \mathrm{~g}$ ). 
Fig. 4 CDK4 plays key roles in the R-point transition. a HEK293 cells were serum-starved for $24 \mathrm{~h}$, stimulated with $10 \%$ serum, and harvested at the indicated time points. Time-dependent formation of the BRD2-RUNX3, E2F1-RUNX3, CDK4-RUNX3, Cyclin D1-RUNX3, HDAC4-RUNX3, p16INK4a_CDK4, p21-CDK4, Cyclin D1-CDK4, and HDAC4-CDK4 complexes was measured by IP and IB. Time-dependent phosphorylation of pRB (at Ser-795) and ERK1/2 was measured by IB. b PLA assay showing the RUNX3-CDK interaction $2 \mathrm{~h}$ after serum stimulation. $\mathbf{c}$ HEK293 cells were treated with control or CDK4specific siRNA (si-con or si-CDK4), serum-starved for $24 \mathrm{~h}$, and then stimulated with serum for the indicated durations. Time-dependent formation of the BRD2-RUNX3, CDK4-RUNX3, HDAC4-RUNX3, and Cyclin D1-RUNX3 complexes and phosphorylated RUNX3 were measured by IP and IB. Time-

dependent expression of ARF was measured by IB. d HEK293 cells were treated with CDK4 inhibitor (PD0332991, 500 nM), serum-starved for 24 h, and then stimulated with serum for the indicated durations. Time-dependent formation of the BRD2-RUNX3 complex was measured by IP and IB. Timedependent expression of ARF was measured by IB. e Cells were serum-starved for $24 \mathrm{~h}$, and then stimulated with $10 \%$ serum. Cells were harvested at the indicated time points. Time-dependent RUNX3-CDK4 interaction and RUNX3 phosphorylation at Ser-356 were measured by IP and IB. f, $\mathbf{g}$ HEK293 cells were transfected with Myc-RUNX3, Myc-RUNX3-S356A, or Myc-RUNX3-S356E, serum-starved for $24 \mathrm{~h}$, and then stimulated with 10\% serum. Cells were harvested at the indicated time points. Time-dependent formation of the BRD2-RUNX3 complex, RUNX3 phosphorylation at Ser-356, and ARF expression were monitored by IP and IB. $\mathbf{h}$ Schematic illustration of the process of Rpa-RX3-AC $\rightarrow$ Rpa-RX3-TR transition. CDK4 of Rpa-RX3-AC and Cyclin D1 of PRC1-Cyclin D1-HDAC4 provide docking sites for the interaction of the two complexes, enabling formation of Rpa-RX3-TR

Inhibition of mTOR, a downstream effecter of the RAS-PI3KAKT pathway, by Rapamycin also maintained Rpa-RX3-AC and prolonged ARF expression for up to $8 \mathrm{~h}$ (Fig. 5h). These results demonstrate that the $\mathrm{PI} 3 \mathrm{~K}$ pathway also contributes to the transition from Rpa-RX3-AC to Rpa-RX3-TR by inducing Cyclin D1. Inhibition of p38 MAPK did not affect association/ dissociation of RUNX3 and BRD2, suggesting that p38 MAPK is not involved in the R-point transition (Supplementary Fig. 6b).

These results demonstrate that the three major pathways downstream of RAS (MEK, JNK, and PI3K) contribute to the Rpoint transition at distinct stages. The contributions of these pathways to each stage of the R-point are summarized in Fig. 6.

Oncogenic K-RAS inhibits the R-point transition. Our results show that the transition from Rpa-RX3-AC to Rpa-RX3-TR occurs only after MEK activity is downregulated (Fig. $4 \mathrm{a}$, pERK1/2). Therefore, we asked what would happen if the RAS-RAF-MEK pathway was constitutively activated. Ectopic expression of oncogenic K-RAS (Myc-K-RASG12V) facilitated the interactions of RUNX3 with p300, BRD2, SWI/SNF, TFIID, and CDK4, and maintained the complex for up to $8 \mathrm{~h}$ (Fig. $7 \mathrm{a}$ ). By contrast, expression of Myc-K-RAS ${ }^{\mathrm{G} 12 \mathrm{~V}}$ inhibited the interactions between RUNX3, Cyclin D1, and HDAC4, as well as the interaction between BRD2 and PRC1 (Fig. 7a). The R-point transition and maintenance of Rpa-RX3-AC by oncogenic K-RAS occurred not only in HEK293 cells, but also in WI-38 human embryonic lung fibroblasts (Supplementary Fig. 7a).

ChIP analysis also showed that expression of oncogenic K-RAS maintained binding of Rpa-RX3-AC to the ARF locus, but inhibited binding of Cyclin D1, HDAC4 and PRC1 (Fig. 7b). The results of ChIP analyses of the $p 21$ promoter yielded essentially the same results as the analogous experiments for $A R F$ (Supplementary Fig. 7b). These results suggest that oncogenic $K-R A S$ facilitates Rpa-RX3-AC formation but inhibits Rpa-RX3TR formation. In addition, oncogenic $K-R A S$ facilitated formation of the PRC1-Cyclin D1 complex (Fig. 7a), but inhibited incorporation of HDAC4 into the complex (Fig. 7a). Therefore, oncogenic $K-R A S$ inhibits Rpa-RX3-TR formation by inhibiting assembly of HDAC4 into the PRC1-Cyclin D1 complex.

ChIP analysis revealed that $\mathrm{H} 4 \mathrm{~K} 12-\mathrm{ac}$ and $\mathrm{H} 3 \mathrm{~K} 4-\mathrm{me} 3$ histone marks were maintained for a long time by expression of Myc-KRAS $^{\mathrm{G} 12 \mathrm{~V}}$ (Fig. 7b and Supplementary Fig. 7b). Consistently, when Myc-K-RASG12V was expressed, ARF and p21 (targets of RpaRX3-AC) were not downregulated, but were instead maintained for long periods (Fig. 7a). Prolonged binding of Rpa-RX3-AC and maintenance of H4K12-ac and H3K4-me3 histone marks at the $A R F$ locus by expression of oncogenic K-RAS were abolished by deletion of the RUNX-binding site from the locus (Fig. 7c).
In addition to oncogenic $K-R A S$, oncogenic $B-R A F$ ( $B$ $R A F^{V 600 E}$ ) also maintained Rpa-RX3-AC, prolonged expression of ARF, and stabilized p53 for long periods (Fig. 7d). These results demonstrate that the $A R F$ locus is opened by Rpa-RX3-AC and closed at the R-point in normal cells, but is not closed in cells expressing oncogenic $K-R A S$ or oncogenic $B-R A F$ due to failure of the Rpa-RX3-AC $\rightarrow$ Rpa-RX3-TR transition.

Exogenously expressed MEK1-CA maintained the CyclinD1-RNF2 interaction but inhibited the CyclinD1-HDAC4 interaction, indicating that MEK1-CA inhibits the R-point transition by inhibiting formation of the PRC1/CyclinD1/ HDAC4 complex (Fig. 7e). An overview of the differential regulation of the R-point by normal RAS and oncogenic RAS is provided in Fig. 7f. An overview of the R-point transition and Rpoint-associated chromatin dynamics in response to normal mitogenic signals is provided in Fig. 8 and Supplementary movie 1.

RUNX3 suppresses oncogenic $K$-RAS-driven oncogenesis. We next investigated the protective role of RUNX3 against endogenous oncogenic K-RAS in $\mathrm{H} 460$ human lung cancer cells (K-RAS-activated without amplification, RUNX3-inactivated, $A R F$ wild-type, and $p 53$ wild-type). By stable transfection, we obtained cell lines expressing H460-vec, H460-ERT2-RUNX3 (expressing ERT2 fused with wild-type RUNX3), and H460ERT2-RUNX3-K94/171R (expressing ERT2 fused with RUNX3 mutant, which does not interact with BRD2 $\left.{ }^{14}\right)$. The ERT2 fusion proteins localized to the nucleus $8 \mathrm{~h}$ after 4 -hydroxytamoxifen (4OHT) treatment (Fig. 9a). In the absence of inducer, none of the cell lines formed Rpa-RX3-AC at any time point after serum stimulation (Fig. 9b), even though they expressed oncogenic $K$ RAS. At $8 \mathrm{~h}$ after inducer treatment, H460-ERT2-RUNX3 cells, but not H460-ERT2-RUNX3-K94/171R cells, formed Rpa-RX3AC (Fig. 9b). In inducer-treated H460-ERT2-RUNX3 cells, RpaRX3-AC was maintained and the ARF-p53 pathway was activated for a long time (Fig. 9b). ChIP analysis confirmed that Rpa-RX3AC bound to its target locus for a long time in inducer-treated H460-ERT2-RUNX3 cells (Fig. 9c). Consistently, H4K12-ac and H3K4-me3 were enriched, whereas H3K27-me3 was diminished, in H460-ERT2-RUNX3 cells, but not in H460-ERT2-RUNX3K94/171R cells, 8-16 h after induction (Fig. 9c). These results indicate that inducer-treated H460-ERT2-RUNX3 cells clearly responded to endogenous oncogenic $K-R A S$ via the R-point defense program, but this process was not initiated in $\mathrm{H} 460$ ERT2-RUNX3-K94/171R cells and parental H460 cells, which lack RUNX3 expression.

Consequently, H460-ERT2-RUNX3 cells, but not H460-ERT2RUNX3-K94/171R cells, underwent apoptosis after inducer 
a

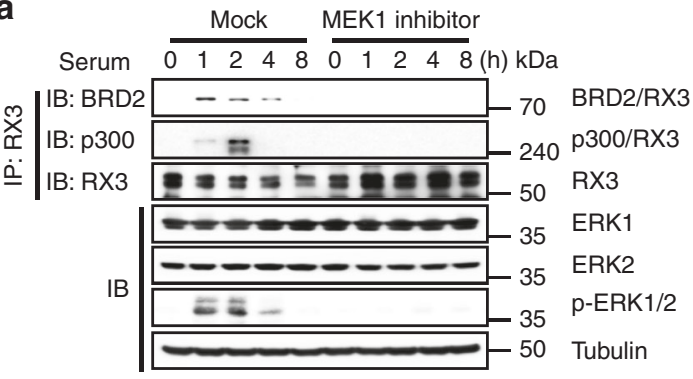

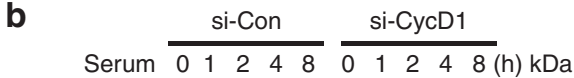

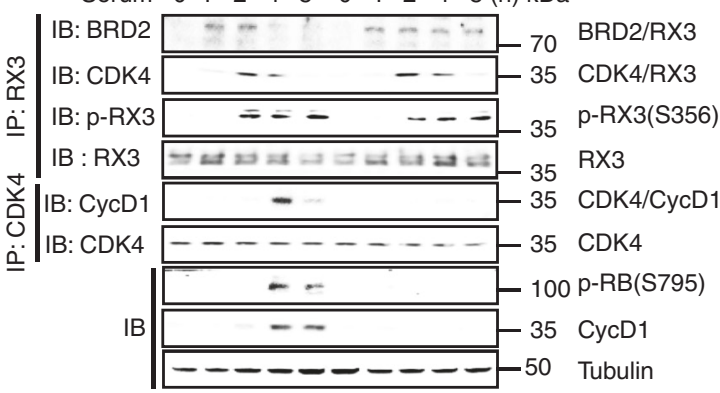

$\begin{array}{llllllll}C & \text { Serum } & 0 & 1 & 2 & 4 & 8 \text { (h) } \mathrm{kDa}\end{array}$

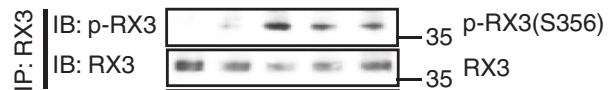

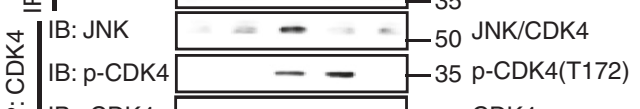

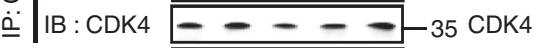

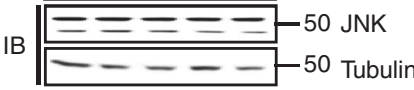

d

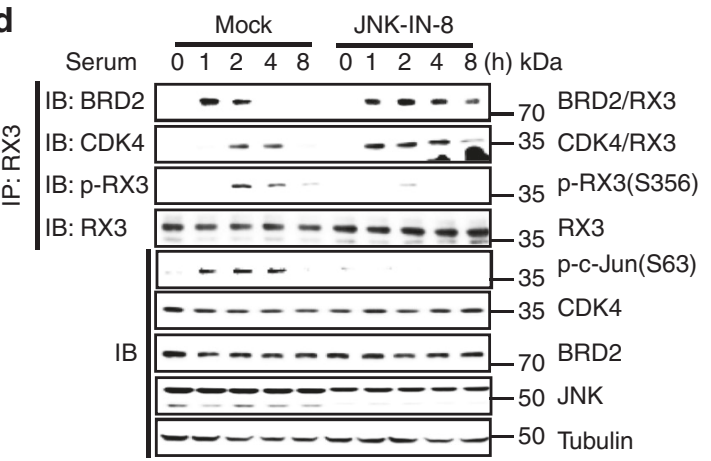

e

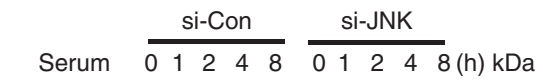

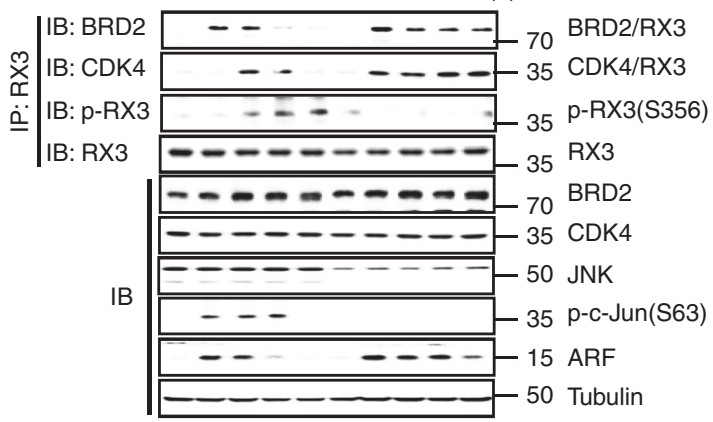

f Flag-CDK4 $\frac{\text { Myc-RX3 }}{\mathrm{WT}}$

Serum $\longdiv { 1 2 4 8 } \overline { 1 2 4 8 }$ (h) kDa

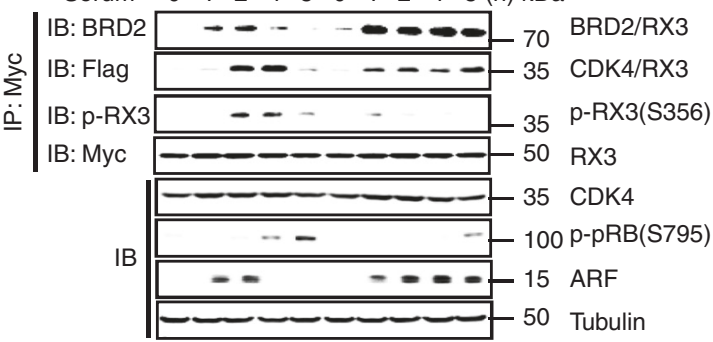

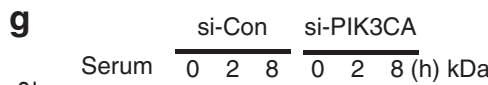

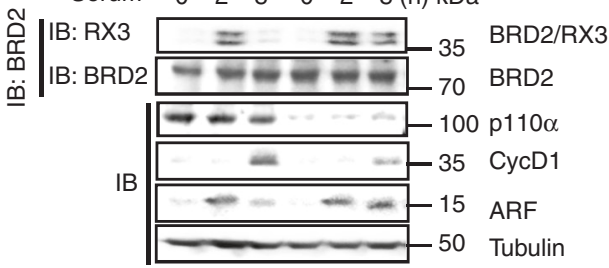

h

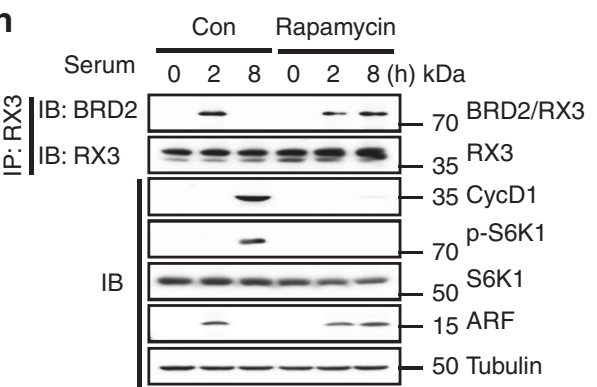

Fig. 5 Multiple signals contribute to the R-point transition. a HEK293 cells were treated with MEK1 inhibitor (U0126, $1 \mu$ M). Time-dependent interactions of BRD2-RUNX3 and p300-RUNX3, as well as phosphorylation of ERK1/2, were monitored by IP and/or IB. b HEK293 cells were treated with control or CyclinD1-specific siRNA (si-con or si-CycD1). Time-dependent formation of the BRD2-RUNX3 and CDK4-RUNX3 complexes and phosphorylation of RUNX3 at Ser-356 and pRB at Ser-795 were measured by IP and IB. c Time-dependent formation of the JNK-CDK4 complexes and phosphorylations of RUNX3 at Ser-356 and CDK4 at Thr-172 were measured by IP and IB. d HEK293 cells were treated with JNK inhibitor (JNK-IN-8, $1 \mu$ M). Time-dependent formation of the BRD2-RUNX3 and RUNX3-CDK4 complexes and phosphorylation of RUNX3 at Ser-356 were measured by IP and IB. Time-dependent expression of ARF was measured by IB. e HEK293 cells were treated with control or JNK-specific siRNA (si-con or si-JNK). Time-dependent formation of the BRD2-RUNX3 and CDK4-RUNX3 complexes and phosphorylation of RUNX3 at Ser-356 were measured by IP and IB. Time-dependent expression of ARF was measured by IB. $\mathbf{f}$ HEK293 cells were transfected with Myc-RUNX3 and Flag-CDK4 WT or Flag-CDK4-T172A (CDK4 mutant defective in phosphorylation by JNK). Time-dependent formation of the BRD2-RUNX3 and CDK4-RUNX3 complexes and phosphorylation of RUNX3 at Ser-356 were measured by IP and IB. Time-dependent phosphorylation of pRB and expression of ARF were measured by IB. $\mathbf{g}$ HEK293 cells were treated with control or PIK3CA-specific siRNA (si-con or si- PIK3CA). Time-dependent formation of the BRD2-RUNX3 complex was measured by IP and IB. Time-dependent expression of ARF was measured by IB. $\mathbf{h}$ HEK293 cells were treated with control or mTORC1 inhibitor (Rapamycin, $100 \mathrm{nM}$ ). Time-dependent formation of the BRD2-RUNX3 complex was measured by IP and IB. Time-dependent expression of ARF was measured by IB. Ribosomal protein S6 kinase beta-1 (S6K1), which is phosphorylated by mTOR signaling, was used for control 


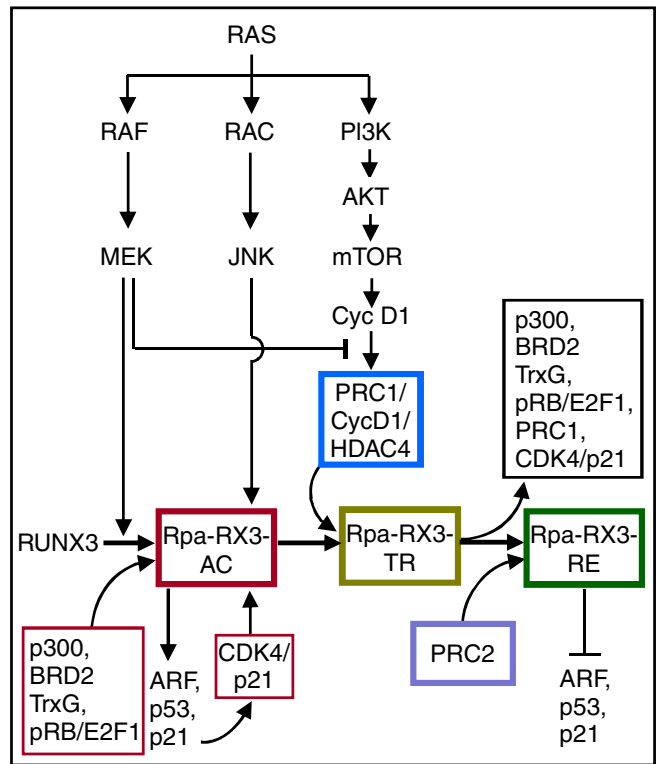

Fig. 6 The roles of the RAS pathway in regulating the R-point transition. The RAS-RAF-MEK pathway facilitates formation of Rpa-RX3-AC and inhibits formation of the PRC1-Cyclin D1-HDAC4 complex. The RAS-RAC-JNK pathway activates CDK4 within Rpa-RX3-AC. The RAS-PI3K pathway facilitates formation of Rpa-RX3-TR by contributing to translation of Cyclin D1

treatment (Supplementary movie 2). FACS analysis at various time points after inducer treatment also revealed that the apoptosis rate of H460-ERT2-RUNX3 cells began to increase $16 \mathrm{~h}$ after inducer treatment, and increased further thereafter (Fig. 9d). Apoptosis was markedly suppressed by siRNAmediated knockdown of p53 (Fig. 9d and Supplementary Fig. 8). Analysis of cell-cycle stage of H460-ER-RX3 cells after 4-OHT treatment revealed that the populations of G1- and S-phase cells were slightly increased and decreased, respectively, by treatment with 4-OHT (Supplementary Fig. 9). Therefore, it appears that the 4-OHT-treated H460-ER-RX3 cells enter the apoptotic pathway from $G_{1}$ phase. H460-vec cells and H460-ERT2RUNX3-K94/171R cells did not undergo apoptosis (Fig. 9d). These results demonstrate that Rpa-RX3-AC defends the cell against endogenous oncogenic $K-R A S$, and that the ARF-p53 pathway is engaged in the R-point defense program.

The R-point governs multiple programs of tumor suppression. To identify genes regulated by Rpa-RX3-AC at the R-point, we performed mRNA sequencing (RNA-seq) in H460-ERT2RUNX3 and H460-ERT2-RUNX3-K94/171R cells. H460-vec cells were used as controls to eliminate any effect of inducer alone. Two-dimensional plots of expression changes in response to inducer treatment revealed that 423 and 362 genes were induced and suppressed, respectively [ $\log _{2}$ (fold change) $\left.\mid \geq 2\right]$, in H460-ERT2-RUNX3 cells $8 \mathrm{~h}$ after inducer treatment (Fig. 10a, red spots). The numbers of genes induced and suppressed increased to 992 and 1221, respectively, after $16 \mathrm{~h}$ (Fig. 10a, blue spots). By contrast, very few genes were affected by inducer treatment in H460-ERT2-RUNX3-K94/171R cells (Fig. 10a). These results suggest that Rpa-RX3-AC mediated most of the upand downregulation of genes in inducer-treated H460-ERT2RUNX3 cells. As RUNX3 does not form the repressor complex (Rpa-RX3-RE) in H460 cells due to the oncogenic K-RAS mutation, the repressed genes were likely indirect targets of RUNX3.
Analysis of the Z-scores of major RUNX3-dependent signaling pathways revealed that genes involved in apoptosis, cell-cycle arrest, inflammatory response, and immune response were induced (Fig. 10b). On the other hand, genes involved in cell proliferation, DNA replication, and metabolic pathways were suppressed by RUNX3 expression (Fig. 10b). These results suggest that the R-point defends against oncogenic $K$-RASinduced tumorigenesis not only by regulating intracellular programs (cell cycle, apoptosis, and metabolic pathways), but also by regulating extracellular programs (inflammatory response and immune response). RUNX3-dependent up- and downregulated genes involved in major signaling pathways are listed in Supplementary Fig. 10. Detailed RNA-seq results are provided in the Excel file H460-RUNX3.xlsx (Supplementary data 1$)$.

\section{Discussion}

The molecular mechanism by which cells regulate R-pointassociated gene expression to make a signal-dependent R-point decision is one of the most fundamental issues in cell biology. In this study, we showed that RUNX3 transactivates R-pointassociated genes by binding to its target loci and opening chromatin structure by sequential recruitment of mediator, chromatin-remodeling complex, basal transcription complex, histone modifiers, and cell-cycle regulators, which together form Rpa-RX3-AC. Therefore, our results identify RUNX3 as a pioneer factor of the R-point and reveal the molecular mechanisms by which appropriate chromatin remodelers and histone modifiers are selectively recruited to target loci in response to mitogenic signals.

Orchestration of gene expression to direct embryonic development includes the TrxG proteins within COMPASS (complex of proteins associated with Set1 ${ }^{28}$. Although both Rpa-RX3-AC and COMPASS contain TrxG proteins, these complexes are distinct. The major differences are that (1) Rpa-RX3-AC is assembled only at target chromatin loci, (2) Rpa-RX3-AC interacts with PcG proteins to form Rpa-RX3-TR at the R-point, and (3) assembly/disassembly of Rpa-RX3-AC is controlled by cellcycle regulators, whereas COMPASS is not. Therefore, identification of RUNX3, a pioneer factor of the R-point, as the core of Rpa-RX3-AC reveals a new mechanism underlying the dynamic regulation of R-point-associated genes.

The emerging picture of chromatin function in cancer involves complex interplay of chromatin-modifying enzymes. In general, loss of TrxG and gain of PcG is a common theme in human cancer, demonstrating the respective tumor-suppressive and oncogenic roles of these proteins ${ }^{29}$. This is also consistent with our claim that the R-point constitutes an oncogene surveillance mechanism: TrxG could suppress tumors through R-point regulation as a component of Rpa-RX3-AC, and PcG could drive cell-cycle progression by destroying Rpa-RX3-AC.

Although TrxG and PcG play opposing roles in transcriptional programs ${ }^{29}$, some promoters are associated with both marks (H3K4-me3 and H3K27-me3), a phenomenon known as bivalent modification $^{30-32}$. For example, $A R F$ is a bivalent gene ${ }^{33}$. It is worth mentioning that most bivalent genes were identified by population analysis in unsynchronized cells. Our results show that the chromatin structure of the $A R F$ locus is dynamically regulated during the cell cycle. Therefore, the chromatin structure of some bivalent genes may be dynamically regulated during the cell cycle.

We previously showed that Runx3 is downregulated in most of $K$-Ras-activated human and mouse lung ADC cells ${ }^{14}$. Our results explain that some $K-R A S$-activated lung ADC cells can proliferate in the absence of RUNX3 because the R-point is deregulated. 
a

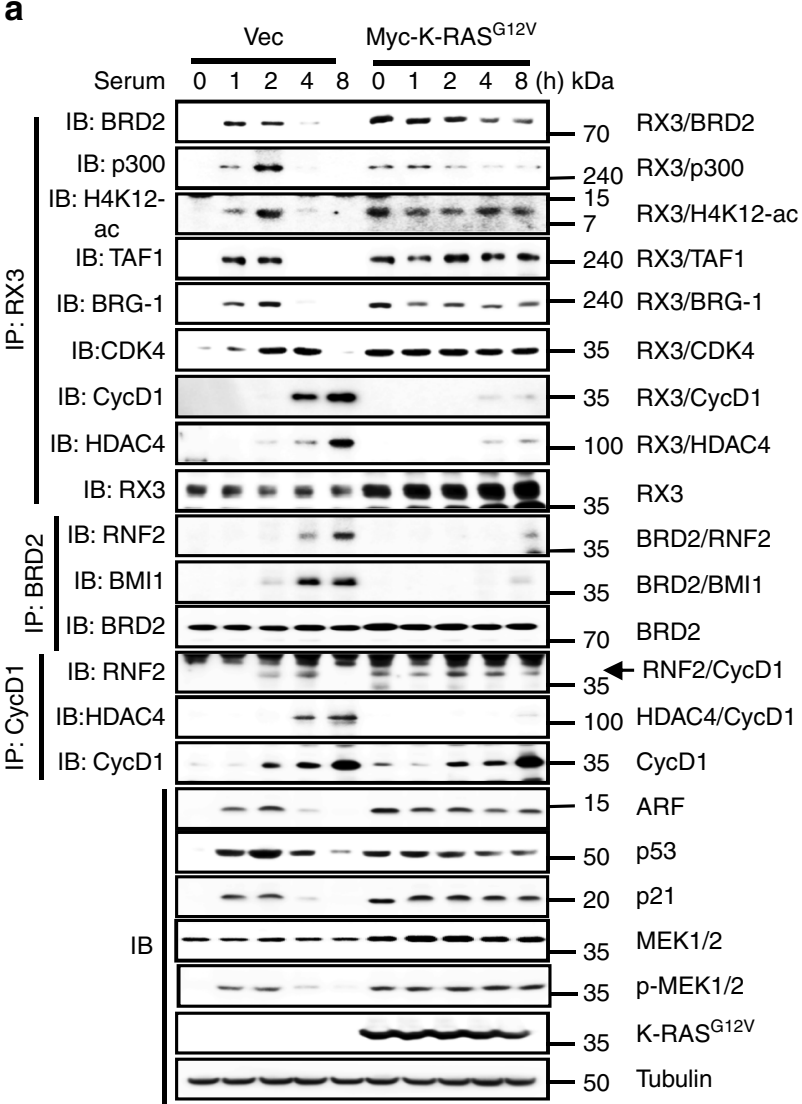

b

志

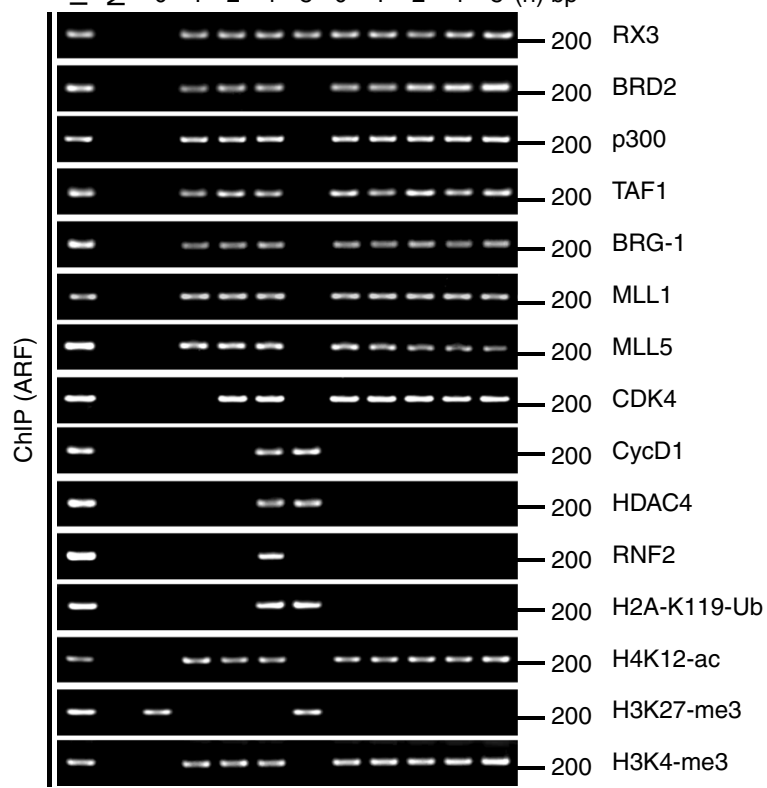

Thus, our results demonstrate that the R-point constitutes an oncogene surveillance mechanism and explain why the R-point is perturbed in nearly all cancer cells. It is worth emphasizing that in multiple kinds of tumors, RUNX3 is frequently inactivated by epigenetic alterations ${ }^{13}$, which could in theory be reversed ${ }^{34}$. Therefore, RUNX3 represents a therapeutic target for multiple types of tumors.
C

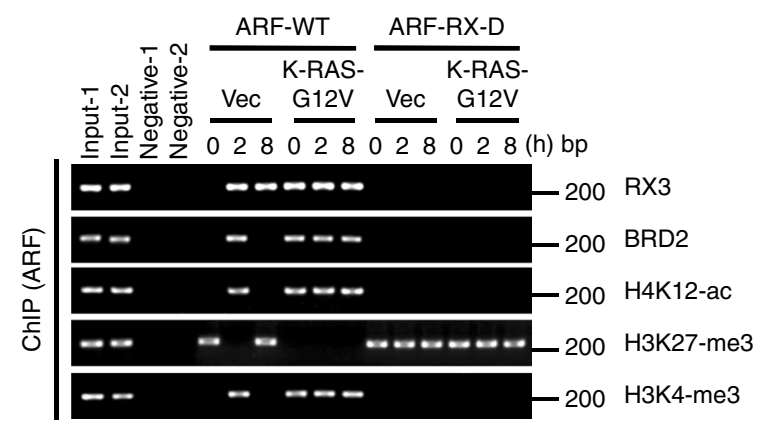

d

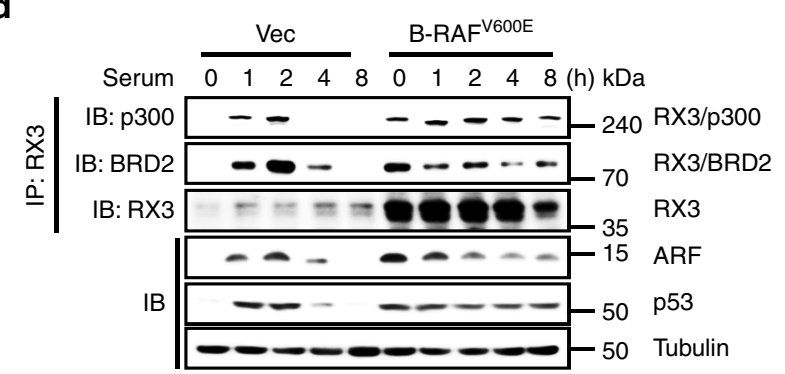

e

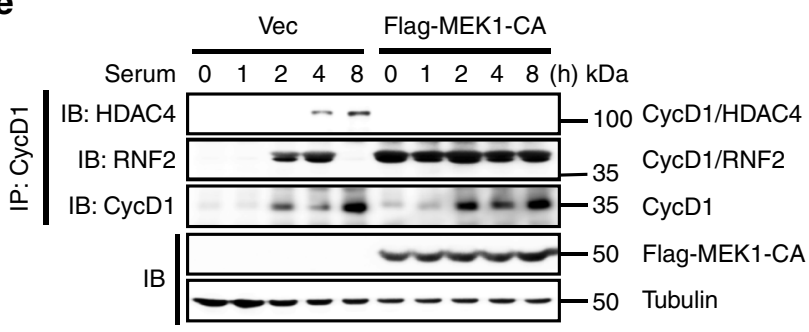

f

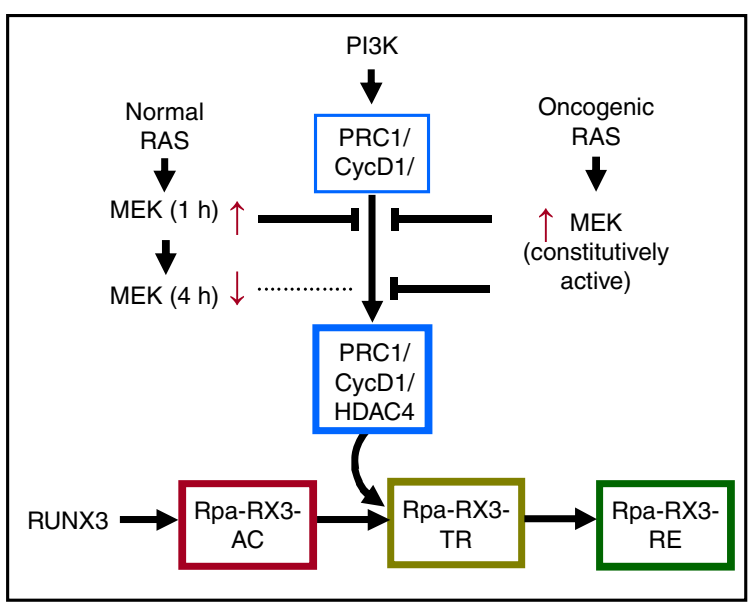

\section{Methods}

Cell lines. HEK293 cells (ATCC, Manassas, VA, USA) were maintained in Dulbecco's modified Eagle's medium (Gibco BRL, Thermo Fisher Scientific, Waltham MA, USA) supplemented with $10 \%$ fetal bovine serum (Gibco BRL), and $1 \%$ penicillin/streptomycin (Invitrogen, Carlsbad, CA, USA). WI-38 cells (Lonza, Basel, Switzerland) were maintained in Dulbecco's modified Eagle's medium (Gibco BRL, Thermo Fisher Scientific, Waltham, MA, USA) supplemented with $10 \%$ fetal bovine serum (Gibco BRL), 1\% MEM Non-Essential Amino Acids 
Fig. 7 R-point surveils aberrant oncogene activation. a HEK293 cells were transfected with empty vector (Vec) or Myc-K-RASG12V. The time-dependent interactions among the components of Rpa-RX3-AC, Rpa-RX3-TR, and Rpa-RX3-RE were measured by IP and IB. Expression levels of ARF, p53, p21, and Myc-K-Ras G12V were measured by IB. b Binding of the components of Rpa-RX3-AC, Rpa-RX3-TR, and Rpa-RX3-RE to the p14ARF promoter and histone marks (H4K12-ac, H3K27-me3, H3K4-me3, and H2A-K119-Ubi) at the locus were measured by ChIP at the indicated time points. One-thirtieth of the lysates were PCR-amplified as input samples. c Wild-type HEK293 cells (HEK293-ARF-WT) and HEK293-ARF-RX-D cells were transfected with empty vector ( $\mathrm{Vec}$ ) or Myc-K-Ras G12V. The binding of RUNX3, BRD2, H4K12-ac, H3K27-me3, and H3K4-me3 to the ARF promoter was measured by ChIP at the indicated time points. One-thirtieth of the lysates were PCR-amplified as input samples. d HEK293 cells were transfected with empty vector (Vec) or B-RAFV600E. Time-dependent formation of the RUNX3-p300 and BRD2-RUNX3 complexes was measured by IP and IB. Time-dependent expression of ARF and p53 was measured by IB. e HEK293 cells were transfected with empty vector (Vec) or Flag-MEK1-CA. Time-dependent interactions of CyclinD1-HDAC4 and CyclinD1-RNF2 were monitored by IP and IB. $\mathbf{f}$ Schematic illustration of differential regulation of the R-point in response to normal and oncogenic RAS. The RAS-RAF-MEK pathway inhibits formation of the PRC1/CyclinD1/HDAC4 complex, and thus inhibits the Rpa-RX3-AC $\rightarrow$ RpaRX3-TR transition. When the RAS-RAF-MEK pathway is activated by mitogenic stimulation, the activated pathway is downregulated after $4 \mathrm{~h}$, allowing PRC1/CyclinD1/HDAC4 complex formation, which is followed by the Rpa-RX3-AC $\rightarrow$ Rpa-RX3-TR transition. If the RAF-MEK pathway is activated by oncogenic RAS, the constitutively activated signal inhibits formation of the PRC1/CyclinD1/HDAC4 complex for a long period of time, thereby inhibiting the Rpa-RX3-AC $\rightarrow$ Rpa-RX3-TR transition

(Gibco BRL) and $1 \%$ penicillin/streptomycin (Invitrogen, Carlsbad, CA, USA). H460 cells (ATCC, Manassas, VA, USA) and H460 stable cell lines were maintained in RPMI 1640 medium (Gibco BRL) supplemented with $10 \%$ fetal bovine serum (Gibco BRL) and 1\% penicillin/streptomycin (Invitrogen). All cell lines were incubated at $37^{\circ} \mathrm{C}$ with $5 \% \mathrm{CO}_{2}$.

Drosophila genetics. GMR-Gal4 was obtained from the Bloomington Drosophila Stock Center (Bloomington, IN, USA). UAS-Lz was kindly provided by U. Banerjee (University of California, Los Angeles, CA, USA) ${ }^{35}$. An EP line harboring an enhancer $\mathrm{P}$ element insertion within the upstream regulatory region of Trithorax (G14137) was obtained from GeneExel (KIST, Daejeon, South Korea). Drosophila stocks were maintained and cultured on standard cornmeal-yeast-agar medium at $25^{\circ} \mathrm{C}$.

DNA transfection, IP, and IB. Transient transfections in all cell lines were performed using Lipofectamine Plus reagent and Lipofectamine (Invitrogen). Cell lysates were incubated with the appropriate mono- or polyclonal antibodies $\left(2 \mu \mathrm{g}\right.$ antibody $/ 500 \mu \mathrm{g}$ lysate sample) for $3 \mathrm{~h}$ at $4{ }^{\circ} \mathrm{C}$, and then with protein G-Sepharose beads (Amersham Pharmacia Biotech, Piscataway, NJ, USA) for $1 \mathrm{~h}$ at $4{ }^{\circ} \mathrm{C}$. For detection of endogenous proteins, lysates were incubated with the appropriate mono- or polyclonal antibodies (dilution range 1:1000-1:3000) for $6-12 \mathrm{~h}$ at $4{ }^{\circ} \mathrm{C}$, and then with protein G-Sepharose beads (Amersham Pharmacia Biotech) for $3 \mathrm{~h}$ at $4{ }^{\circ} \mathrm{C}$. Immunoprecipitates were resolved on SDS-polyacrylamide gel electrophoresis (SDS-PAGE) gels and transferred to a polyvinylidene difluoride membrane (Millipore, Billerica, MA, USA). The membrane was immunoblotted with the appropriate antibodies after blocking and visualized on an Amersham ${ }^{\text {w }}$ Imager 600 (GE Healthcare, Chicago, IL, USA) after treatment with ECL solution (Amersham Pharmacia Biotech).

Antibodies. Antibodies targeting Cyclin D1 (Cat\# sc-20044), CDK4 (Cat\# sc-260), HDAC4 (Cat\# sc-11418), p-c-Jun (Cat\# SC-822), p-ATF (Cat\# SC-8398), p110 (Cat\# SC-7174), p300 (Cat\# sc-584), p53 (Cat\# sc-126), p21 (Cat\# sc-397), p14 (Cat\# sc-8340, Cat\# sc-53640), ERK1 (Cat\# SC-94), ERK1/2 (Cat\# SC-135900), E2F1 (Cat\# sc-137059), TAF1 (Cat\# sc-735), TAF7 (Cat\# sc-292282), TBP (Cat\# sc-421), BRG-1 (Cat\# sc-17796, Cat\# sc-10768), and BAF155 (Cat\# sc-10756) were obtained from Santa Cruz Biotechnology (Dallas, TX, USA). All antibodies of Santa Cruz Biotechnology were diluted to 1:1000. Antibodies targeting H2AK119-ub (Cat\# 8240 S), H3K412-ac (Cat\# 2591 S), H3K27-me3 (Cat\# 9733 S), H3K4-me3 (Cat\# 9751 S), RNF2 (Cat\# 5694 S), BMI1 (Cat\# 6964 S), EZH2 (Cat\# 5246 S), phospho-pRB(Ser-795) (Cat\# 9301 S), phospho-ERK1/2 (Cat\# 9101 S), JNK (Cat\# 92525), S6K (Cat\# 2708 S), p-S6K (Cat\# 9234 S), p38 MAPK (Cat\# 9212 S) and Acetylated Lys (Cat\# 9441 L) were obtained from Cell Signaling Technology (Danvers, MA, USA). All antibodies of Cell Signaling Technology were diluted to 1:1000. Antibodies targeting RUNX3(5G4) (Cat\# ab40278), p16 (Cat\# ab108349) and EED (Cat\# ab4469) were obtained from Abcam (Cambridge, UK). All antibodies of Abcam were diluted to 1:3000. Antibodies targeting HA (12CA5; dilution 1:1000; Cat\# 11666606 001, Roche Applied Science, Mannheim, Germany), FLAG (M2; dilution 1:3000; Cat\# F1804, Sigma, MO, USA), Myc (9E10; dilution 1:1000; Cat\# sc-40, Santa Cruz Biotechnology), BRD2 (M01; dilution 1:1000; Cat\# H00006046-M01, Abnova, Taipei City, Taiwan), pRB (dilution 1:1000; Cat\# 554136, BD Biosciences, CA, USA), p-CDK4 (dilution 1:1000; Cat\# PA564482, Invitrogen, CA, USA), MLL5 (dilution 1:1000; Cat\# STJ27895, St. John's Laboratory, London, UK) and MLL1 (dilution 1:1000; Cat\# A300-374A, Bethyl Laboratories Inc., TX, USA) were used for IB and IP. Anti RUNX3-phospho-S356 (dilution 1:1000) was made rabbit polyclonal anti-serum against synthetic RUNX3 peptide phosphorylated at Ser-356.
In vitro translation. The $\mathrm{TnT}^{\circledast}$ Coupled Reticulocyte Lysate System (Promega, Madison, WI, USA) is available in two configurations, for transcription and translation of genes cloned downstream of either the T7 or SP6 RNA polymerase promoters. To use these systems, $2.0 \mu \mathrm{g}$ of circular plasmid DNA containing a

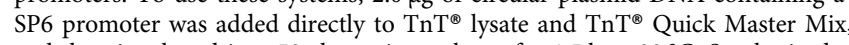
and then incubated in a $50 \mu \mathrm{l}$ reaction volume for $1.5 \mathrm{~h}$ at $30^{\circ} \mathrm{C}$. Synthesized proteins were analyzed by SDS-PAGE.

Proximity ligation assay (PLA). The PLA was performed using the Duolink ${ }^{\circledast}$ In Situ PLA ${ }^{\circledR}$ Kit (Sigma, St. Louis, MO, USA). Briefly, cells were grown, fixed, and permeabilized. The samples were then incubated overnight at $4^{\circ} \mathrm{C}$ with primary antibodies against the two proteins to be examined, washed [Buffer A: $0.01 \mathrm{M}$ Tris$\mathrm{HCl}(\mathrm{pH} 7.4), 0.15 \mathrm{M} \mathrm{NaCl}$, and $0.05 \%$ Tween 20], incubated at $37^{\circ} \mathrm{C}$ for $60 \mathrm{~min}$ with specific probes, stained for F-actin to visualize cytoplasm, and washed with Buffer B [0.2 M Tris- $\mathrm{HCl}(\mathrm{pH} 7.5)$ and $0.1 \mathrm{M} \mathrm{NaCl}$. Signals were visualized as distinct fluorescent spots on a fluorescence microscope (Carl Zeiss AXIO Zoom. V16 and ApoTome.2). Background correction, contrast adjustment of raw images, and quantification of fluorescence signals were performed using the Zen 2012 Blue Edition software (Carl Zeiss, Oberkochen, Germany).

Inhibitor and siRNA. CDK4 inhibitor (PD0332991), JNK inhibitor (JNK-IN-8), MEK1 inhibitor (U0126), p38 MAPK inhibitor (SB203580) and Rapamycin (R8781) were purchased from Sigma-Aldrich. Cells were treated with CDK4 inhibitor $(500 \mathrm{nM})$, JNK inhibitor $(1 \mu \mathrm{M})$, MEK1 inhibitor $(1 \mu \mathrm{M})$, p38 MAPK inhibitor $(1 \mu \mathrm{M})$ or Rapamycin $(100 \mathrm{nM})$, and harvested at the indicated time points after serum stimulation. Knockdown analysis was performed by transfecting HEK293 cells with $50 \mathrm{nM}$ siRNA using RNAiMAX (Invitrogen, CA, USA) before serum starvation. Cells were harvested at the indicated time points after serum stimulation. BRD2, p53, CDK4, RNF2, and Cyclin D1 siRNAs were purchased from Bioneer (Daejeon, South Korea). HDAC4 siRNAs was purchased from Cell Signaling Technology. Sequences of siRNAs were as follows:

(si-BRD2 sense: CACUUGGCCUGCAUGACUA

antisense: UAGUCAUGCAGGCCAAGUG)

(si-p53 sense: CAGUUUGAGGUGCGUGUU

antisense: AACACGCACCUCAAAGCUG)

(si-CDK4 sense: CCAGAAUCUACAGCUACCA

antisense: UGGUAGCUGUAGAUUCCUGG)

(si-Cyclin D1 sense: GACCUUCGUUGCCCUCUGU

antisense: ACAGAGGGCAACGAAGGUC)

(si-RNF2 sense: UGAUAGGGUAUUGAGUGUA

antisense: UACACUCAAUACCCUAUCA)

Deletion of the RUNX-binding site in ARF enhancer region. To delete the RUNX-binding site (GACCGCA) in the CDKN2A $\left(p 14^{A R F}\right)$ enhancer region (ntd -1466) by the CRISPR/Cas9 method, HEK293 cells were transfected with the pRGEN-CDKN2A target plasmid (target sequence: 5'-GACGGATCCAGGCAGA CCGCAGG) and pRGEN-Cas9-Hyg-CMV (ToolGen, Seoul, South Korea). The cells were maintained in standard culture medium (10\% Dulbecco's modified Eagle's medium) containing $800 \mu \mathrm{g} / \mathrm{ml}$ hygromycin B (H3274; Sigma). Hygromycin B-resistant cells were selected. Deletion of only the RUNX3-binding site in the ARF promoter region was confirmed by nucleotide sequencing.

ChIP assay. ChIP assays were performed using the ChIP assay kit (cat \# 17-295; Millipore). HEK293 cells or H460-derived stable cell lines were serum-starved for $24 \mathrm{~h}$, treated with $10 \%$ serum or $10 \%$ serum $/ 1 \mu \mathrm{M} 4-\mathrm{OHT}$ (Sigma), harvested at the indicated time points, and cross-linked with formaldehyde $(1 \%[\mathrm{v} / \mathrm{v}] ,10 \mathrm{~min}$, $37^{\circ} \mathrm{C}$ ). Chromatin was immunoprecipitated with the indicated antibodies. The p14 


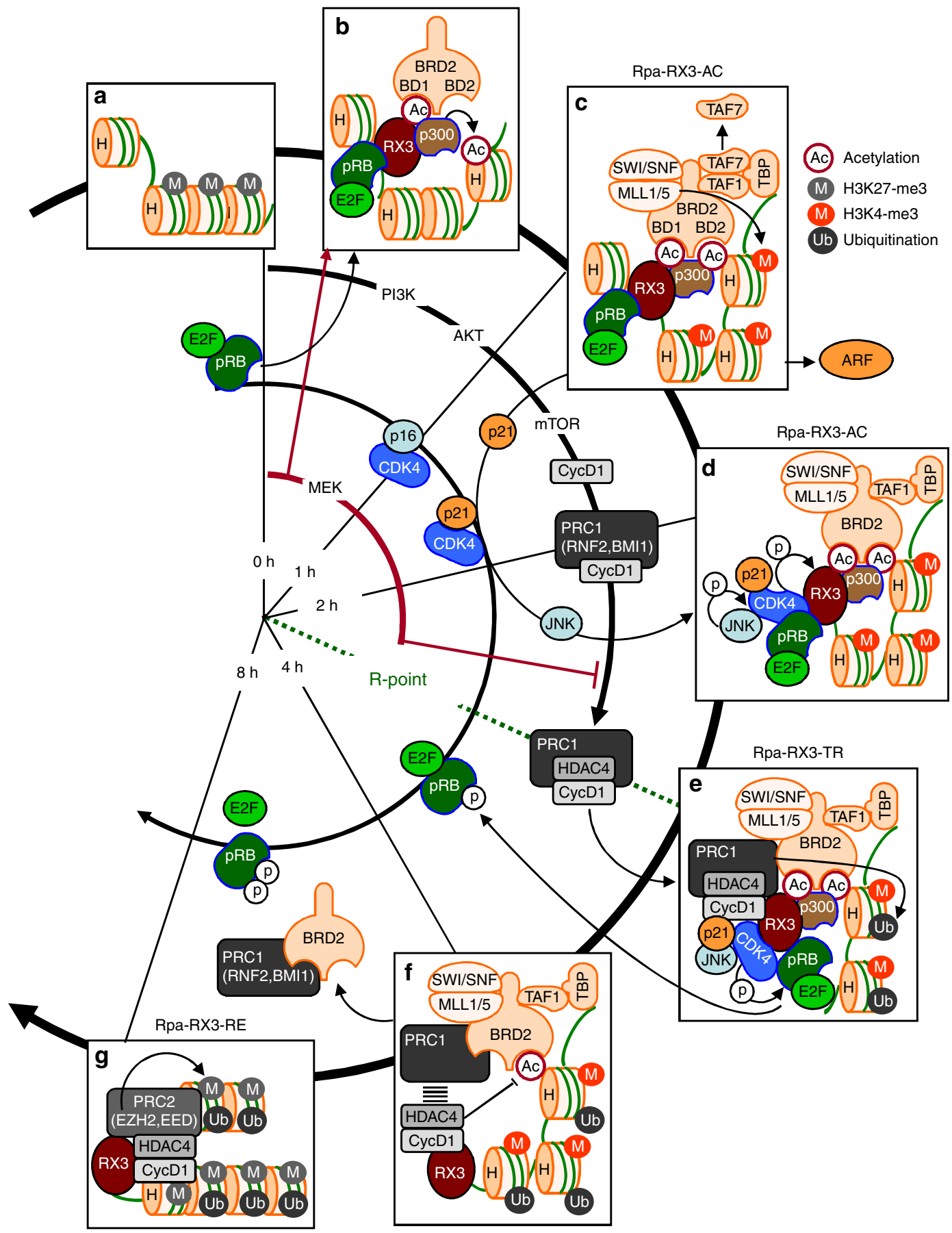

Fig. 8 The sequential molecular events for the R-point decision. $\mathbf{a}, \mathbf{b}$ Upon mitogenic stimulation, RUNX3 binds to inactive chromatin marked by H3K27-me3. pRB-E2F1 and p300 associate with RUNX3. p300 acetylates RUNX3 and histones. BRD2 binds to acetylated RUNX3 through its first bromodomain (BD1). c One hour after mitogenic stimulation, the second bromodomain (BD2) of BRD2 binds to H4K12-ac: BRD2 binds both acetylated RUNX3 and acetylated histone through its bromodomains. Subsequently, SWI/SNF, MLL1/5, and TFIID bind to the C-terminal region of BRD2. At this point, inhibitory histone marks (H3K27me3) are erased, and activatory marks (H3K4-me3) are enriched at the locus. Soon thereafter, TAF7 (inhibitory TAF) is released from the large complex, and expression of ARF, p53, and p21 is induced. The large complex, of which RUNX3 is the core, was named as Rpa-RX3-AC. d Two hours after mitogenic stimulation, CDK4 (associated with p21) binds to RUNX3 and becomes an additional component of Rpa-RX3-AC. At this point, the Cyclin D1-PRC1 complex forms separately from Rpa-RX3-AC. e When the RAS-MEK signal is downregulated, the Cyclin D1-PRC1 complex matures into the Cyclin D1-HDAC4-PRC1 complex, which in turn binds to Rpa-RX3-AC through the interaction between Cyclin D1 and CDK4 (a component of Rpa-RX3-AC), yielding Rpa-RX3-TR. Activation of CDK4 through the association with Cyclin D1 is critical for the inactivation of the chromatin loci and the dissociation of the entire complex. If the RAS signal is constitutively activated, the Cyclin D1-PRC1 complex fails to mature into the Cyclin D1-HDAC4-PRC1 complex, and consequently cannot form Rpa-RX3-TR. Therefore, if R-point commitment is normal, cells expressing constitutively active RAS cannot progress through the R-point into S-phase. $\mathbf{f}$ If the mitogenic signal is downregulated in a normal manner, Rpa-RX3-TR dissociates (4 h after stimulation) into two pieces, RUNX3-Cyclin D1-HDAC4 and BRD2-PRC1-SWI/SNF-TFIID, which remain associated with chromatin. $\mathbf{g}$ Soon thereafter, EZH2 associates with RUNX3-Cyclin D1-HDAC4 to form Rpa-RX3$\mathrm{RE}$, which remains on the chromatin. EZH2 contributes to the enrichment of an inactive chromatin mark (H3K27-me3) at the locus 
a

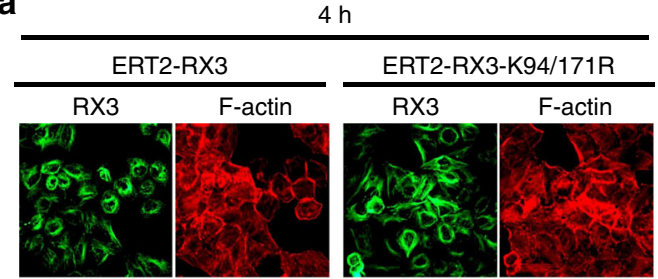

$8 \mathrm{~h}$

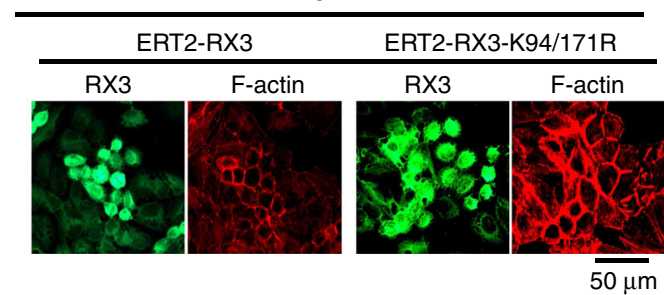

b

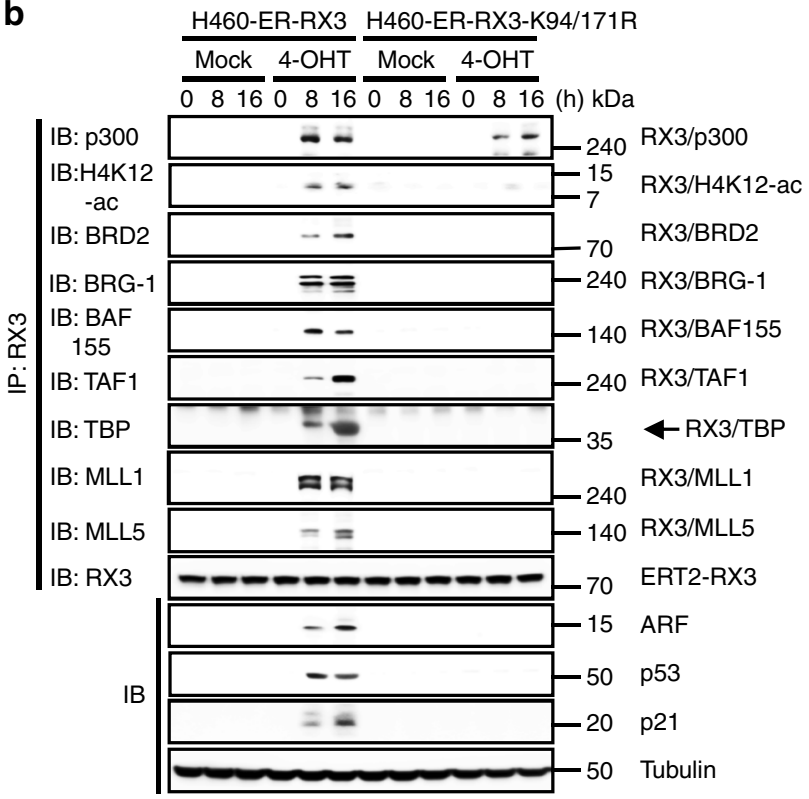

C

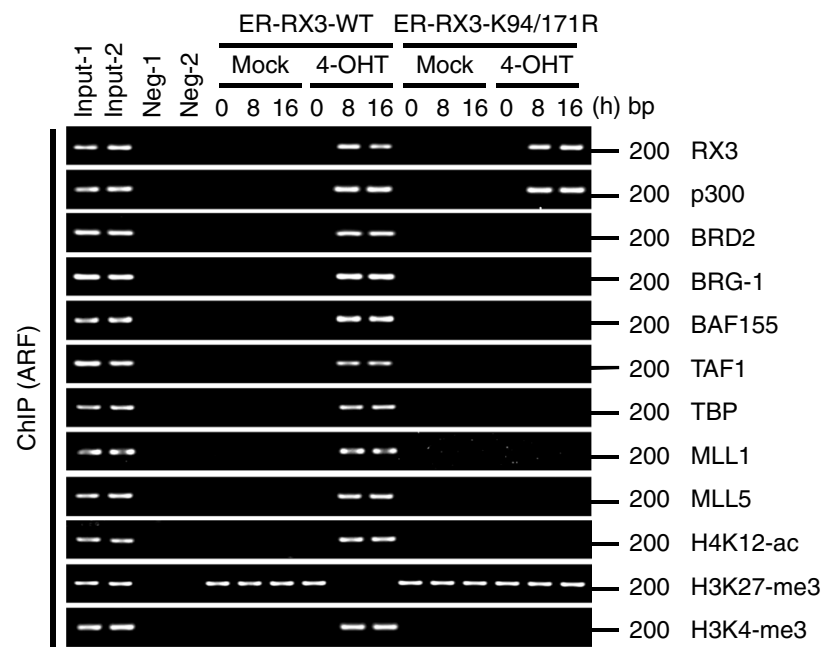

d

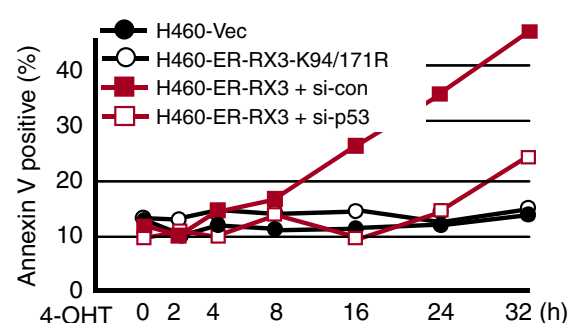

$\begin{array}{llllllll}4-\mathrm{OHT} & 0 & 2 & 4 & 8 & 16 & 24 & 32(\mathrm{~h})\end{array}$

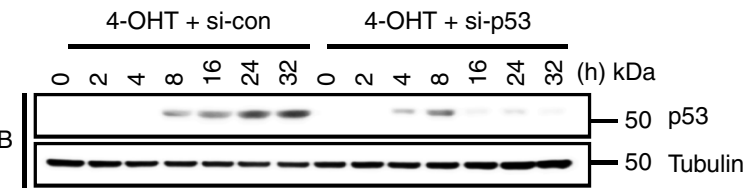

Fig. 9 RUNX3 defends against endogenous oncogenic K-Ras. a H460-ERT2-RUNX3 and H460-ERT2-RUNX3-K94/171R cells were synchronized by serum deprivation and stimulated with $10 \%$ serum and $1 \mu \mathrm{M} 4-\mathrm{OHT}$ for the indicated durations $(0,4$, and $8 \mathrm{~h})$. Time-dependent subcellular localization of the expressed proteins was analyzed by double immunofluorescence staining (green = RUNX3; red = F-actin). b, c H460-ERT2-RUNX3 and H460-ERT2RUNX3-K94/171R cells were serum-starved for $24 \mathrm{~h}$, stimulated with $10 \%$ serum or $10 \%$ serum $+1 \mu \mathrm{M} 4-\mathrm{OHT}$. Cells were harvested at the indicated time points, and the time-dependent interactions of RUNX3 with BRD2, p300, H4K12-ac, TFIID complex (TAF1, TAF7, and TBP), SWI/SNF complex (BRG-1 and BAF155), and MLL1/5 were measured by IP and IB. Expression of p14ARF, p53, and p21 was measured by IB. The binding of the proteins and H4K12-ac, H3K27-me3, and H3K4-me3 to the ARF promoter was measured by ChIP at the indicated time points. One-thirtieth of the lysates were PCR-amplified as input samples. d H460-vec, H460-ERT2-RUNX3, and H460-ERT2-RUNX3-K94/171R cells were treated with indicated si-RNA, serum-starved for 24 h, and then stimulated with $10 \%$ serum or $10 \%$ serum $+1 \mu \mathrm{M} 4-\mathrm{OHT}$ for the indicated durations. Apoptotic cells were detected by flow cytometry after Annexin $\mathrm{V}$-FITC/PI staining. The levels of p53 were measured by IB

promoter region was amplified by PCR using the following primers (previously reported primers ${ }^{20}$ )

( $p 14^{A R F}$-Forward: AGTGGCTACGTAAGAGTGATCGC)

( $p 14^{A R F-R e v e r s e: ~ C T T A C A G A T C A G A C G T C A A G C C C) ~}$

Chromosome conformation capture assay (3C assay). Briefly, $1.0 \times 10^{7}$ cells were cross-linked using $2 \%$ formaldehyde in $10 \mathrm{ml}$ of phosphate-buffered saline (PBS) containing $10 \%$ fetal bovine serum and incubated for $10 \mathrm{~min}$ at room temperature. The reaction tubes were quenched with $1.425 \mathrm{ml}$ of $1 \mathrm{M}$ glycine. The fixed cells were washed twice with ice-cold PBS and then harvested using $1 \mathrm{ml}$ of ice-cold PBS. Harvested cells were re-suspended in $5 \mathrm{ml}$ of cold lysis buffer [10 mM Tris- $\mathrm{HCl}(\mathrm{pH} 7.5), 10 \mathrm{mM} \mathrm{NaCl}, 0.2 \% \mathrm{NP}-40$, and protease inhibitors] and incubated for $10 \mathrm{~min}$ on ice. The lysates were centrifuged at $400 \mathrm{~g}$ for $10 \mathrm{~min}$ at $4{ }^{\circ} \mathrm{C}$. The pelleted nuclei were washed with $0.5 \mathrm{ml}$ of $1.2 \times$ restriction enzyme buffer, and then re-suspended in $0.5 \mathrm{ml}$ of the same buffer. SDS was added to a final concentration of $0.3 \%$, and pelleted nuclei were incubated for $1 \mathrm{~h}$ at $37^{\circ} \mathrm{C}$. Triton X-100 was added to a final concentration of $2 \%$ to quench the SDS, and the nuclei were incubated for $1 \mathrm{~h}$ at $37^{\circ} \mathrm{C}$. Next, $400 \mathrm{U}$ of $\mathrm{XbaI}$ was added, and the sample was incubated overnight at $37^{\circ} \mathrm{C}$. SDS was added to a concentration of $1.6 \%$, and the sample was incubated for $25 \mathrm{~min}$ at $65^{\circ} \mathrm{C}$. Digested genomic DNA was suspended in $6.125 \mathrm{ml}$ of ice-cold $1.15 \times$ ligation buffer containing $1 \%$ Triton X-100. T4 DNA ligase (100 U) was added, and the reaction mixtures were incubated for $4 \mathrm{~h}$ at $16^{\circ} \mathrm{C}$, followed by $30 \mathrm{~min}$ at room temperature. Reaction mixtures were then treated with $300 \mu \mathrm{g}$ of proteinase $\mathrm{K}$ at $65^{\circ} \mathrm{C}$ overnight. DNA was purified by the phenol-chloroform method. Purified DNA was dissolved in $150 \mu \mathrm{l}$ of $10 \mathrm{mM}$ Tris (pH 7.5). DNA was amplified by PCR using the following primers.

(3C assay Primer A-Forward: GGCGCCAGGCCGGGTCGA)

(3C assay Primer B-Reverse: TCGCGTCCCCGCTCCCCTATT)

(3C assay Primer C-Forward: CAGCCTCCTGATTGGCGGATAG)

(3C assay Primer D-Reverse: CCACCATCTTCCCACCCTCAG) 
a

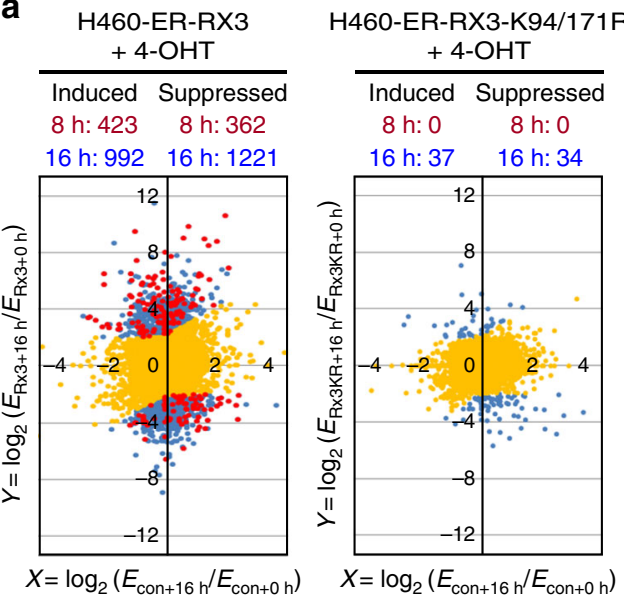

b

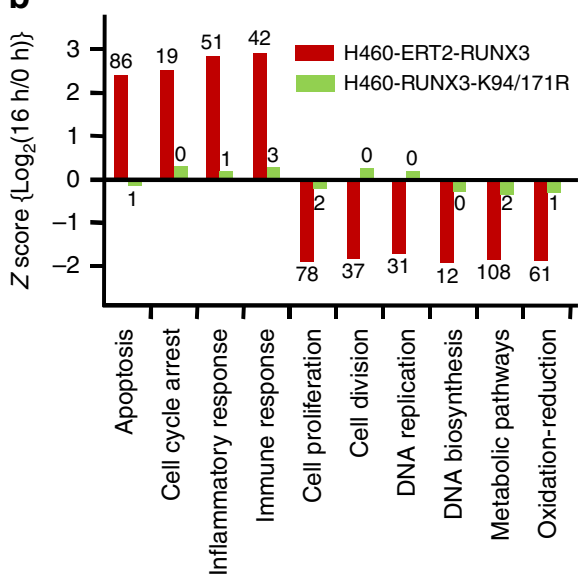

Fig. 10 The R-point governs multiple programs of tumor suppression. a H460-vec, H460-ERT2-RUNX3, and H460-ERT2-RUNX3-K94/171R cells were serum-starved for $24 \mathrm{~h}$, and then stimulated with $10 \%$ serum or $10 \%$ serum $+1 \mu \mathrm{M} \mathrm{4-OHT}$ for 0 , 8 , or $16 \mathrm{~h}$. RNA was extracted from the cells, and gene expression patterns were analyzed by mRNA sequencing. Expression of genes 8 or $16 \mathrm{~h}$ after serum stimulation was quantified as log ${ }_{2}$ (fold change) relative to the average of control reactions (i.e., before serum stimulation, $0 \mathrm{~h}$ ) for each cell line. Differential gene expression in response to expression of wild-type RUNX3 was analyzed by plotting $\log _{2}\left(E_{\mathrm{R} \times 3+16 \mathrm{~h}} / E_{\mathrm{R} \times 3+\mathrm{O}}\right)$ and $\log _{2}\left(E_{\mathrm{vec}+16 \mathrm{~h}} / E_{\mathrm{vec}+\mathrm{Oh}}\right)$; the results are shown on the left. Differential gene expression

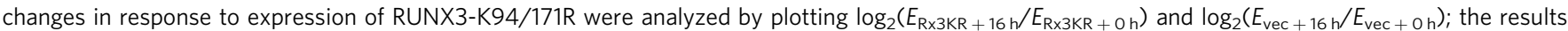
are shown on the right. $E_{\mathrm{R} \times 3}+16 \mathrm{~h}$ and $E_{\mathrm{R} \times 3+0 \mathrm{~h}}, E_{\mathrm{R} \times 3 K R+16 \mathrm{~h}}$ and $E_{\mathrm{R} \times 3 K R}+\mathrm{O} \mathrm{h}$, and $E_{\mathrm{vec}-16 \mathrm{~h}}$ and $E_{\mathrm{vec}-\mathrm{o}} \mathrm{h}$ are the average expression levels of genes 0 or $16 \mathrm{~h}$ after 4-OHT stimulation in H460-ERT2-RUNX3, H460-ERT2-RUNX3-K94/171R, and H460-vec cells, respectively. Yellow spots indicate genes regulated in a RUNX3-independent manner. Red and blue spots indicate genes regulated in a RUNX3-dependent manner 8 and $16 \mathrm{~h}$ after $4-\mathrm{OHT}$ stimulation, respectively (FDR $<0.001, p<0.05)$. b RUNX3-dependent genes involved in various signaling pathways were analyzed using the DAVID Bioinformatics Resources $6.8^{37}$. Gene expression levels $16 \mathrm{~h}$ after $4-\mathrm{OHT}$ stimulation in the indicated cells were quantified as the fold change relative to the average of unstimulated levels. Z-scores of $\log _{2}\left(E_{\mathrm{R} \times 3}+16 \mathrm{~h} / E_{\mathrm{R} \times 3}+\mathrm{O} h\right)$ and $\log _{2}\left(E_{\mathrm{R} \times 3 \mathrm{KR}}+16 \mathrm{~h} / E_{\mathrm{R} \times 3 \mathrm{KR}+\mathrm{O}}\right)$ for major signaling pathways are shown for $\mathrm{H} 460-\mathrm{ERT} 2-\mathrm{RUNX3}$ and H460-ERT2-RUNX3-K94/171R cells. Numbers at the top or bottom of the bars indicate the numbers of genes significantly up- or downregulated by inducer treatment. All categories were enriched with $p<0.05$

Yeast two-hybrid screening. Yeast two-hybrid screening was carried out using the Matchmaker Gold Yeast Two-Hybrid system (Clontech/Takara, Mountain View, CA, USA). The C-terminal region of BRD2 (aa 450-802) was used as bait. Prey proteins were expressed from the universal human cDNA library (Cat. No. 630481;Clontech/Takara). Bait- and prey-transformed yeast (strain Y187) were mated, and the resultant diploids were cultured in DDO (SD-Leu/-Trp) medium to select for the presence of both plasmids. Subsequently, the diploids were cultured in $\mathrm{DDO} / \mathrm{X} / \mathrm{A}$ (SD-Leu/-Trp/X-a-gal/ABA) and QDO/X/A (SD-Leu/-Trp/-His/-Ade/ $\mathrm{X}$ - $\alpha$-gal/ABA) medium to select diploids exhibiting protein-protein interactions. Tenfold serial dilutions were performed prior to colony plating in order to ensure that growth on the selective medium was dependent solely on HIS3, ADE2, ABA, and MEL1 reporter gene expression. Selected colonies were subjected to DNA sequencing. Leu, leucine; Trp, tryptophan; His, histidine; Ade, adenine; SD, synthetic defined medium; ABA, aureobasidin A (antibiotic).

Flow cytometry assay. Cells were harvested and processed using the FITC-Annexin V Apoptosis Detection Kit I (BD Biosciences, San Jose, CA, USA) and propidium iodide DNA staining protocol. Cell apoptosis and cell cycle were analyzed by flow cytometry on a BD FACSCalibur machine (BD Biosciences). All of data were determined using the Flowjo software (https://www.flowjo.com).

RNA-seq analysis. Isolated total RNA was processed for preparation of an RNAseq library using the Illumina TruSeq Stranded mRNA Sample Preparation kit (Illumina, San Diego, CA, USA). Quality and size of libraries were assessed using the Agilent 2100 Bioanalyzer DNA kit (Agilent, Santa Clara, CA, USA). All libraries were quantified by qPCR using a CFX96 Real Time System (Bio-Rad, Hercules, CA, USA) and sequenced on NextSeq500 sequencers (Illumina). Sequencing adapters and low-quality bases in the raw reads were trimmed using the Cutadapt software. The cleaned high-quality reads were mapped to the human reference genome hg19 (https://genome.ucsc.edu) using STAR software. Genes differentially expressed between two selected biological conditions were identified by Cuffdiff in the Cufflinks package (http://cole-trapnell-lab.github.io/cufflinks/papers/).

Quantification and statistical analysis. For cell line studies, heatmaps were analyzed by the log-rank test using the PermutMatrix software ${ }^{36}$. Gene clustering was analyzed using DAVID Bioinformatics Resources $6.8^{37}$.

Reporting summary. Further information on research design is available in the Nature Research Reporting Summary linked to this article.

\section{Data availability}

A reporting summary for this Article is available as a Supplementary Information file. The RNA-seq data are available under accession GSE128174 at Gene Expression Omnibus (GEO) and figshare with the identifier (https://doi.org/10.6084/m9. figshare.7775777.v1 ${ }^{38}$ ). The source data for Figures and Supplementary Figures are provided as a Source Data file. All relevant data are available from the corresponding author.

Received: 24 August 2018 Accepted: 2 April 2019

Published online: 23 April 2019

\section{References}

1. Malumbres, M. \& Barbacid, M. To cycle or not to cycle: a critical decision in cancer. Nat. Rev. Cancer 1, 222-231 (2001).

2. Pardee, A. B. A restriction point for control of normal animal cell proliferation. Proc. Natl Acad. Sci. USA 71, 1286-1290 (1974).

3. Weinberg, R. A. The biology of cancer. In: Elizabeth Z (ed.) 275-329 (Garland Science, New York, 2014).

4. Chi, X. Z. et al. Runx3 plays a critical role in restriction-point and defense against cellular transformation. Oncogene 36, 6884-6894 (2017).

5. Zaret, K. S. \& Carroll, J. S. Pioneer transcription factors: establishing competence for gene expression. Genes Dev. 25, 2227-2241 (2011).

6. Jozwik, K. M. \& Carroll, J. S. Pioneer factors in hormone-dependent cancers. Nat. Rev. Cancer 12, 381-385 (2012).

7. Soufi, A. et al. Pioneer transcription factors target partial DNA motifs on nucleosomes to initiate reprogramming. Cell 161, 555-568 (2015).

8. Sekiya, T. \& Zaret, K. S. Repression by Groucho/TLE/Grg proteins: genomic site recruitment generates compacted chromatin in vitro and impairs activator binding in vivo. Mol. Cell 28, 291-303 (2007).

9. Strahl, B. D. \& Allis, C. D. The language of covalent histone modifications. Nature 403, 41-45 (2000)

10. Clapier, C. R., Iwasa, J., Cairns, B. R. \& Peterson, C. L. Mechanisms of action and regulation of ATP-dependent chromatin-remodelling complexes. Nat. Rev. Mol. Cell Biol. 18, 407-422 (2017).

11. Wang, $\mathrm{H}$. et al. Role of histone $\mathrm{H} 2 \mathrm{~A}$ ubiquitination in Polycomb silencing. Nature 431, 873-878 (2004) 
12. Cao, R. et al. Role of histone H3 lysine 27 methylation in Polycomb-group silencing. Science 298, 1039-1043 (2002).

13. Ito, Y., Bae, S. C. \& Chuang, L. S. The RUNX family: developmental regulators in cancer. Nat. Rev. Cancer 15, 81-95 (2015).

14. Lee, Y. S. et al. Runx 3 inactivation is a crucial early event in the development of lung adenocarcinoma. Cancer Cell 24, 603-616 (2013).

15. Zetterberg, A., Larsson, O. \& Wiman, K. G. What is the restriction point? Curr. Opin. Cell Biol. 7, 835-842 (1995).

16. Huang, $\mathrm{H}$. et al. Solution structure of the second bromodomain of Brd2 and its specific interaction with acetylated histone tails. BMC Struct. Biol. 7, 57 (2007).

17. LeRoy, G., Rickards, B. \& Flint, S. J. The double bromodomain proteins Brd2 and Brd3 couple histone acetylation to transcription. Mol. Cell 30, 51-60 (2008).

18. Denis, G. V. et al. Identification of transcription complexes that contain the double bromodomain protein $\mathrm{Brd} 2$ and chromatin remodeling machines. J. Proteome Res. 5, 502-511 (2006).

19. Wang, L. et al. The leukemogenicity of AML1-ETO is dependent on sitespecific lysine acetylation. Science 333, 765-769 (2011).

20. Linggi, B. et al. The $t(8 ; 21)$ fusion protein, AML1 ETO, specifically represses the transcription of the p14(ARF) tumor suppressor in acute myeloid leukemia. Nat. Med. 8, 743-750 (2002).

21. Hagege, H. et al. Quantitative analysis of chromosome conformation capture assays (3C-qPCR). Nat. Protoc. 2, 1722-1733 (2007).

22. Jin, Y. H. et al. Transforming growth factor-beta stimulates p300-dependent RUNX3 acetylation, which inhibits ubiquitination-mediated degradation. J. Biol. Chem. 279, 29409-29417 (2004).

23. LaBaer, J. et al. New functional activities for the p21 family of CDK inhibitors. Genes Dev. 11, 847-862 (1997).

24. Cheng, M. et al. The p21(Cip1) and p27(Kip1) CDK 'inhibitors' are essential activators of cyclin D-dependent kinases in murine fibroblasts. EMBO J. 18, 1571-1583 (1999).

25. Zhang, M. et al. PTHrP prevents chondrocyte premature hypertrophy by inducing cyclin-D1-dependent Runx2 and Runx3 phosphorylation, ubiquitylation and proteasomal degradation. J. Cell Sci. 122, 1382-1389 (2009).

26. Kato, J. Y., Matsuoka, M., Strom, D. K. \& Sherr, C. J. Regulation of cyclin D-dependent kinase 4 (cdk4) by cdk4-activating kinase. Mol. Cell Biol. 14, 2713-2721 (1994).

27. Colleoni, B. et al. JNKs function as CDK4-activating kinases by phosphorylating CDK4 and p21. Oncogene 36, 4349-4361 (2017).

28. Piunti, A. \& Shilatifard, A. Epigenetic balance of gene expression by Polycomb and COMPASS families. Science 352, aad9780 (2016).

29. Mills, A. A. Throwing the cancer switch: reciprocal roles of polycomb and trithorax proteins. Nat. Rev. Cancer 10, 669-682 (2010).

30. Azuara, V. et al. Chromatin signatures of pluripotent cell lines. Nat. Cell Biol. 8, 532-538 (2006).

31. Bernstein, B. E. et al. A bivalent chromatin structure marks key developmental genes in embryonic stem cells. Cell 125, 315-326 (2006).

32. Voigt, P., Tee, W. W. \& Reinberg, D. A double take on bivalent promoters. Genes Dev. 27, 1318-1338 (2013).

33. $\mathrm{Li}, \mathrm{H}$. et al. The Ink4/Arf locus is a barrier for iPS cell reprogramming. Nature 460, 1136-1139 (2009).

34. Balmain, A. Cancer: new-age tumour suppressors. Nature 417, 235-237 (2002).

35. Tracey, W. D. Jr., Ning, X., Klingler, M., Kramer, S. G. \& Gergen, J. P. Quantitative analysis of gene function in the Drosophila embryo. Genetics 154, 273-284 (2000).

36. Caraux, G. \& Pinloche, S. PermutMatrix: a graphical environment to arrange gene expression profiles in optimal linear order. Bioinformatics 21, 1280-1281 (2005).
37. Huang da, W., Sherman, B. T. \& Lempicki, R. A. Systematic and integrative analysis of large gene lists using DAVID bioinformatics resources. Nat. Protoc. 4, 44-57 (2009).

38. Lee, J. W. H460-RUNX3.xlsx. https://doi.org/10.6084/m9.figshare.7775777.v1 (2019).

\section{Acknowledgements}

S.-C. Bae is supported by a Creative Research Grant (2014R1A3A2030690) through the National Research Foundation (NRF) of Korea. J.-W. Lee is supported by Basic Science Research Program grant 2018R1C1B6001532, Y.-S. Lee is supported by Basic Science Research Program grant 2017R1D1A3B03034076, J.-W. Jang is supported by Basic Science Research Program grant 2018R1C1B6009179, and J.-W. Hyun is supported by Basic Research Laboratory Program grant NRF-2017R1A4A1014512 through the NRF, funded by the Ministry of Science, ICT and future Planning (MSIP) of Korea government. D.-M. Kim was supported by Korean Foundation of Cancer Research (KFCR-2016-003). I.-Y. Park was supported by "Cooperative Research Program for Agriculture Science and Technology Development (PJ01226101)” Rural Development Administration, Republic of Korea. Y. Ito is supported by the National Research Foundation Singapore and the Singapore Ministry of Education under its Research Centers of Excellence initiative.

\section{Author contributions}

J.W.L. and D.M.K. analyzed assembly and disassembly of the R-point-associated complexes. J.W.J. analyzed fly genetics. Y.S.L., X.Z.C., I.Y.P., and J.W.H. performed yeast two-hybrid screening. T.G.P. and S.H.S. performed the 3 C assay. Y.I. and S.C.B. interpreted the results and wrote the manuscript. All authors contributed to the editing of the manuscript.

\section{Additional information}

Supplementary Information accompanies this paper at https://doi.org/10.1038/s41467019-09810-w.

Competing interests: The authors declare no competing interests.

Reprints and permission information is available online at http://npg.nature.com/ reprintsandpermissions/

Journal peer review information: Nature Communications thanks the anonymous reviewers for their contribution to the peer review of this work. Peer reviewer reports are available.

Publisher's note: Springer Nature remains neutral with regard to jurisdictional claims in published maps and institutional affiliations.

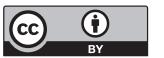

Open Access This article is licensed under a Creative Commons Attribution 4.0 International License, which permits use, sharing, adaptation, distribution and reproduction in any medium or format, as long as you give appropriate credit to the original author(s) and the source, provide a link to the Creative Commons license, and indicate if changes were made. The images or other third party material in this article are included in the article's Creative Commons license, unless indicated otherwise in a credit line to the material. If material is not included in the article's Creative Commons license and your intended use is not permitted by statutory regulation or exceeds the permitted use, you will need to obtain permission directly from the copyright holder. To view a copy of this license, visit http://creativecommons.org/ licenses/by/4.0/.

(C) The Author(s) 2019 